A slightly different version of this manuscript will appear in Mathematical Physics, Analysis

and Geometry.

\title{
GROUPOIDS, VON NEUMANN ALGEBRAS AND THE INTEGRATED DENSITY OF STATES
}

\author{
DANIEL LENZ, NORBERT PEYERIMHOFF, AND IVAN VESELIĆ
}

\begin{abstract}
We study spectral properties of random operators in the general setting of groupoids and von Neumann algebras. In particular, we establish an explicit formula for the canonical trace of the von Neumann algebra of random operators and define an abstract density of states.

While the treatment applies to a general framework we lay special emphasis on three particular examples: random Schrödinger operators on manifolds, quantum percolation and quasi crystal Hamiltonians. For these examples we show that the distribution function of the abstract density of states coincides with the integrated density of states defined via an exhaustion procedure.
\end{abstract}

\section{INTRODUCTION}

The aim of this paper is to review and present a unified treatment of basic features of random (Schrödinger) operators using techniques from Connes' noncommutative integration theory and von Neumann algebras Con79]. Particular emphasis will be laid on an application of the general setting to the example of

- a group action on a manifold proposed by two of the authors [PV01].

This example merges and extends two situations, viz periodic operators on manifolds as studied first by Adachi/Sunada AS93 and random Schrödinger operators on $\mathbb{R}^{d}$ or $\mathbb{Z}^{d}$ as studied by various people (s. below) starting with the work of Pastur Pas71. In the first situation a key role is played by the geometry of the underlying manifold. In the second situation, the crucial ingredient is the randomness of the corresponding potential.

We also apply our discussion to two more examples:

- random operators on tilings and Delone sets whose mathematically rigorous study goes back to Hof [Hof93] and Kellendonk [Kel95]).

- random operators on site-percolation graphs, see e.g. dGLM59a, $\mathrm{CCF}^{+} 86, \mathrm{BK} 01, \mathrm{Ves} 05 \mathrm{~b}$.

As for the above three examples let us already point out the following differences: in the first example the underlying geometric space is continuous and the group acting on it is discrete; in the second example the underlying geometric space is discrete and the group acting on it is continuous; finally, in the third

Date: October 27, 2018, LPV-2007-01-04.tex.

1991 Mathematics Subject Classification. 46L10, 35J10; 46L51, 82B44.

Key words and phrases. Groupoids, von Neumann algebras, integrated density of states, random operators, Schrödinger operators on manifolds, trace formula. 
example both the underlying geometric space and the group acting on it are discrete.

The use of von Neumann algebras in the treatment of special random operators is not new. It goes back at least to the seminal work of Šubin on almost periodic operators [Sub79]. These points will be discussed in more detail next.

Random Schrödinger operators arise in the quantum mechanical treatment of disordered solids. This includes, in particular, periodic operators, almost periodic operators and Anderson type operators on $\mathbb{Z}^{d}$ or $\mathbb{R}^{d}$ (cf. the textbooks [CFSK87, Kir89, CL90, PF92, Sto01]). In all these cases one is given a family $\left(H_{\omega}\right)$ of selfadjoint operators $H_{\omega}$ acting on a Hilbert space $\mathcal{H}_{\omega}$, indexed by $\omega$ in a measure space $(\Omega, \mu)$ and satisfying an equivariance condition with respect to a certain set of unitary operators $\left(U_{i}\right)_{i \in I}$.

While specific examples of these cases exhibit very special spectral features, there are certain characteristics shared by all models. These properties are as follows. (In parentheses we give a reference where the corresponding property is established.)

(P1) Almost sure constancy of the spectral properties of $H_{\omega}$ given some ergodicity condition. In particular, the spectrum $\Sigma$ is nonrandom (Theorem 5.1).

(P2) Absence of discrete spectrum (Corollary 5.9) and, in fact, a dichotomy (between zero and infinity) for the values of the dimensions of spectral projections.

(P3) A naturally arising von Neumann algebra (Section 3) with a canonical trace $\tau$, to which the random operators are affiliated (Section 4).

(P4) A measure $\rho$, called the density of states, governing global features of the family $\left(H_{\omega}\right)$, in particular, having $\Sigma$ as its support (Proposition 5.2). This measure is related to the trace of the von Neumann algebra.

Let us furthermore single out the following point, which we show for the three abovementioned examples:

(P5) A local procedure to calculate $\rho$ via an exhaustion given some amenability condition. This is known as Pastur-Šbin trace formula. It implies the self-averaging property of the density of states (discussed for the examples mentioned above in Sections 6, 7, 8).

Let us now discuss these facts for earlier studied models. The interest in property (P5) arouse from the physics of disordered media. First mathematically rigorous results on the (integrated) density of states are due to Pastur [Pas71, Pas72, Pas74, Fukushima, Nakao and Nagai Fuk74, FNN75, FN77, Nak77, Fuk81, Kotani [Kot77, and Kirsch and Martinelli KM82a, KM82c. In these papers two different methods for constructing the integrated density of states (IDS) can be found (property (P5)). Either one uses the Laplace transform to conclude the convergence of certain normalized eigenvalue counting functions, or one analyzes the counting functions directly via the so called Dirichlet-Neumann bracketing. In our setting the Laplace transform method 
seems to be of better use, since the pointwise superadditive ergodic theorem [AK81] used in the Dirichlet-Neumann bracketing approach [KM82a] has no counterpart in the (nonabelian) generality we are aiming at.

For the more recent development in the study of the IDS of alloy type and related models, as well as the results on its regularity and asymptotic behaviour, see [CL90, PF92, Sto01, Ves04] and the references cited there.

For almost periodic differential operators on $\mathbb{R}^{d}$ and the associated von Neumann algebras, a thorough study of the above features (and many more) has been carried out in the seminal papers by Coburn, Moyer and Singer CMS73. and Šubin [ك̌ Šb79]. Almost periodic Schrödinger operators on $\mathbb{Z}^{d}$ and $\mathbb{R}^{d}$ were then studied by Avron and Simon AS82, AS83.

An abstract $C^{*}$-algebraic framework for the treatment of almost periodic operators was then proposed and studied by Bellissard [Bel86, Bel92] and Bellissard, Lima and Testard [BLT86]. While these works focus on K-theory and the so called gap-labeling, they also show (P1)-(P5) for almost periodic Schrödinger type operators on $\mathbb{R}^{d}$ and $\mathbb{Z}^{d}$. Let us emphasize that large parts of this $C^{*}$ algebraic treatment are not confined to almost periodic operators. In fact, (P1)-(P4) are established there for crossed products arising from arbitrary actions of locally compact abelian groups on locally compact spaces $X$.

After the work of Aubry/André $\mathrm{AA}$ and the short announcement of Bellissard/Testard in [BT82, investigations in this framework, centered around so called spectral duality, were carried out by Kaminker and Xia [KX87] and Chojnacki [Cho92]. A special one-dimensional version of spectral duality based on GJLS99] can also be found in [Len99].

An operator algebraic framework of crossed-products (involving von Neumann crossed products) can also be used in the study of general random operators if one considers $\mathbb{R}^{d}$ actions together with operators on $L^{2}\left(\mathbb{R}^{d}\right)$ (cf. [Len99]). However, certain of these models rather use actions of $\mathbb{Z}^{d}$ together with operators on $L^{2}\left(\mathbb{R}^{d}\right)$, like the thoroughly studied alloy or continuous Anderson type models. This presents a difficulty which was overcome in a work by Kirsch Kir85 introducing a so called suspension construction, see also BLT86, for related material. This allows to "amplify" these $\mathbb{Z}^{d}$ actions to $\mathbb{R}^{d}$ actions and thus reduce the treatment of (P1)-(P4) in the $\mathbb{Z}^{d}$ case to the $\mathbb{R}^{d}$ case.

In recent years three more classes of examples have been considered. These are random operators on manifolds Szz89, Szn90, PV01, LPV04, LPV03, discrete random operators on tilings Hof93, Hof95, Kel95, Kel97, BHZ00, LS01, LS04, and random Hamiltonians generated by percolation processes [BK01, Ves05a, Ves05b, KM]. In these cases the algebraic framework developed earlier could not be used to establish (P1)-(P5). Note, however, that partial results concerning, e.g., (P1) or restricted versions of (P5) are still available. Note also that continuous operators associated with tilings as discussed in BHZ00, BBG01] fall within the $C^{*}$-algebraic framework of [Bel86, Bel92]. A more detailed analysis of the point spectrum of discrete operators associated to tilings and percolation graphs will be carried out in [LV]. 
The model considered in [PV01] includes periodic operators on manifolds. In fact, it was motivated by work of Adachi and Sunada AS93, who establish an exhaustion construction for the IDS as well as a representation as a $\Gamma$-trace in the periodic case. For further investigations related to the IDS of periodic operators in both discrete and continuous geometric settings, see e.g. [DM97, DM98, MY02, MSY03, DLMSY03, LV].

More precisely, our first example concerned with Random Schrödinger operators on Manifolds (RSM) can be described as follows,see [PV01, LPV04]:

Example. (RSM) Let $\left(X, g_{0}\right)$ be the Riemannian covering of a compact Riemannian manifold $M=X / \Gamma$. We assume that there exists a family $\left(g_{\omega}\right)_{\omega \in \Omega}$ of Riemannian metrics on $X$ which are parameterized by the elements of a probability space $\left(\Omega, \mathcal{B}_{\Omega}, \mathbb{P}\right)$ and which are uniformly bounded by $g_{0}$, i.e., there exists a constant $A \geq 1$ such that

$$
\frac{1}{A} g_{0}(v, v) \leq g_{\omega}(v, v) \leq A g_{0}(v, v) \text { for all } v \in T X \text { and } \omega \in \Omega .
$$

Let $\lambda^{\omega}$ denote the Riemannian volume form corresponding to the metric $g_{\omega}$. We assume that $\Gamma$ acts ergodically on $\Omega$ by measure preserving transformations. The metrics are compatible in the sense that for all $\gamma \in \Gamma$ the corresponding deck transformations

$$
\gamma:\left(X, g_{\omega}\right) \rightarrow\left(X, g_{\gamma \omega}\right)
$$

are isometries. Then the induced maps $U_{(\omega, \gamma)}: L^{2}\left(X, \lambda^{\gamma^{-1} \omega}\right) \rightarrow L^{2}\left(X, \lambda^{\omega}\right)$, $\left(U_{(\omega, \gamma)} f\right)(x)=f\left(\gamma^{-1} x\right)$ are unitary operators. Based on this geometric setting, we consider a family $\left(H_{\omega}: \omega \in \Omega\right), H_{\omega}=\Delta_{\omega}+V_{\omega}$, of Schrödinger operators satisfying the following equivariance condition

$$
H_{\omega}=U_{(\omega, \gamma)} H_{\gamma^{-1} \omega} U_{(\omega, \gamma)}^{*},
$$

for all $\gamma \in \Gamma$ and $\omega \in \Omega$. We also assume some kind of weak measurability in $\omega$, namely, we will assume that

$$
\omega \mapsto\left\langle f(\omega, \cdot), F\left(H_{\omega}\right) f(\omega, \cdot)\right\rangle_{\omega}:=\int_{X} \bar{f}(\omega, x)\left[F\left(H_{\omega}\right) f\right](\omega, x) d \lambda^{\omega}(x)
$$

is measurable for every measurable $f$ on $\Omega \times X$ with $f(\omega, \cdot) \in L^{2}\left(X, \lambda^{\omega}\right), \omega \in \Omega$, and every function $F$ on $\mathbb{R}$ which is uniformly bounded on the spectra of the $H_{\omega}$. Note that $L^{2}\left(X, \lambda^{\omega}\right)$ considered as a set of functions (disregarding the scalar product) is independent of $\omega$. The expectation with respect to the measure $\mathbb{P}$ will be denoted by $\mathbb{E}$.

This example covers the following two particular cases:

(I) A family of Schrödinger operators $\left(\Delta+V_{\omega}\right)_{\omega \in \Omega}$ on a fixed Riemannian manifold $\left(X, g_{0}\right)$ with random potentials, see [PV01]. In this case the equivariance condition (11) transforms into the following property of the potentials

$$
V_{\gamma \omega}(x)=V_{\omega}\left(\gamma^{-1} x\right)
$$


(II) A family of Laplacians $\Delta_{\omega}$ on a manifold $X$ with random metrics $\left(g_{\omega}\right)_{\omega \in \Omega}$ satisfying some additional assumptions [LPV04].

By the properties of $X$ and $M$ in (RSM), the group $\Gamma$ is discrete, finitely generated and acts cocompactly, freely and properly discontinuously on $X$.

In the physical literature the equivariance condition (1) is denoted either as equivariance condition, see e.g. [Bel86], or as ergodicity of operators [Pas80, Kir89], where it is assumed that the measure preserving transformations are ergodic. From the probabilistic point of view this property is simply the stationarity of an operator valued stochastic process.

It is our aim here to present a groupoid based approach to $(\mathrm{P} 1)-(\mathrm{P} 4)$ covering all examples studied so far. This includes, in particular, the case of random operators on manifolds, the tiling case and the percolation case.

Our framework applies also to Schrödinger operators on hyperbolic space (e.g. the Poissonian model considered in [Szn89]). However, our proof of (P5) does not apply to this setting because of the lack of amenability of the isometry group.

For the example (RSM) with amenable group action $\Gamma$, we will prove (P5) in Section 6. Note that case (I) of (RSM) includes the models treated earlier by the suspension construction. Thus, as a by product of our approach, we get an algebraic treatment of (P5) for these models. As mentioned already, our results can also be applied to further examples. Application to tilings is discussed in LS01, LS04. There, a uniform ergodic type theorem for tilings along with a strong version of Pastur-Šubin-formula (P5) is given. The results also apply to random operators on percolation graphs. In particular they provide complementary information to the results in [Ves05a, Ves05b, KM], where the integrated density of states was defined rigorously for site and edge percolation Hamiltonians. A basic discussion of these examples and the connection to our study here is given in Sections 7 and 8 respectively. This will in particular show that (P5) remains valid for these examples. For further details we refer to the cited literature. The results also apply to random operators on foliations (see Kor95] for related results).

Our approach is based on groupoids and Connes theory of noncommutative integration Con79]. Thus, let us conclude this section by sketching the main aspects of the groupoid framework used in this article. The work Con79 on noncommutative integration theory consists of three parts. In the first part an abstract version of integration on quotients is presented. This is then used to introduce certain von Neumann algebras (viz. von Neumann algebras of random operators) and to classify their semifinite normal weights. Finally, Connes studies an index type formula for foliations. We will be only concerned with the first two parts of [Con79].

The starting point of the noncommutative integration is the fact that certain quotients spaces (e.g., those coming from ergodic actions) do not admit a nontrivial measure theory, i.e., there do not exist many invariant measurable 
functions. To overcome this difficulty the inaccessible quotient is replaced by a nicer object, a groupoid. Groupoids admit many transverse functions, replacing the invariant functions on the quotient. In fact, the notion of invariant function can be further generalized yielding the notion of random variable in the sense of [Con79]. Such a random variable consists of a suitable bundle together with a family of measures admitting an equivariant action of the groupoid. This situation gives rise to the so called von Neumann algebra of random operators and it turns out that the random operators of the form $\left(H_{\omega}\right)$ introduced above are naturally affiliated to this von Neumann algebra. Moreover, each family $\left(H_{\omega}\right)$ of random operators gives naturally rise to many random variables in the sense of Connes. Integration of these random variables in the sense of Connes yields quite general proofs for main features of random operators. In particular, an abstract version of the integrated density of states is induced by the trace on the von Neumann algebra.

Acknowledgments: It is a pleasure to thank W. Kirsch and P. Stollmann for various stimulating discussions on random operators and hospitality at Ruhr-Universität Bochum, respectively TU Chemnitz. This work was supported in part by the DFG through the SFB 237, the Schwerpunktprogramm 1033, and grants Ve 253/1 \& Ve 253/2 within the Emmy-Noether Programme.

\section{Abstract Setting for basic Geometric objeCts: GROUPOIDS AND RANDOM VARIABLES}

In this section we discuss an abstract generalization for the geometric situation given in example (RSM). The motivation for this generalization is that it covers many different settings at once, such as tilings, percolation, foliations, equivalence relations and our concrete situation, a group acting on a metric space.

Let us first introduce some basic general notations which are frequently used in this paper. For a given measurable space $(S, \mathcal{B})$ we denote the set of measures by $\mathcal{M}(S)$ and the corresponding set of measurable functions by $\mathcal{F}(S)$. The symbol $\mathcal{F}^{+}(S)$ stands for the subset of nonnegative measurable functions. $M_{f}$ denotes the operator of multiplication with a function $f$.

We begin our abstract setting with a generalization of the action of the group $\Gamma$ on the measurable space $\left(\Omega, \mathcal{B}_{\Omega}\right)$. This generalization, given by $\mathcal{G}=\Omega \times \Gamma$ in the case at hand, is called a groupoid. The main reason to consider it is the fact that it serves as a useful substitute of the quotient space $\Omega / \Gamma$, which often is a very unpleasant space (e.g., in the case when $\Gamma$ acts ergodically). The general definition of a groupoid is as follows [Ren80].

Definition 2.1. A triple $\left(\mathcal{G}, \cdot,{ }^{-1}\right)$ consisting of a set $\mathcal{G}$, a partially defined associative multiplication $\cdot$, and an inverse operation ${ }^{-1}: \mathcal{G} \rightarrow \mathcal{G}$ is called a groupoid if the following conditions are satisfied:

- $\left(g^{-1}\right)^{-1}=g$ for all $g \in \mathcal{G}$,

- If $g_{1} \cdot g_{2}$ and $g_{2} \cdot g_{3}$ exist, then $g_{1} \cdot g_{2} \cdot g_{3}$ exists as well, 
- $g^{-1} \cdot g$ exists always and $g^{-1} \cdot g \cdot h=h$, whenever $g \cdot h$ exists,

- $h \cdot h^{-1}$ exists always and $g \cdot h \cdot h^{-1}=g$, whenever $g \cdot h$ exists.

A given groupoid $\mathcal{G}$ comes along with the following standard objects. The subset $\mathcal{G}^{0}=\left\{g \cdot g^{-1} \mid g \in \mathcal{G}\right\}$ is called the set of units. For $g \in \mathcal{G}$ we define its range $r(g)$ by $r(g)=g \cdot g^{-1}$ and its source by $s(g)=g^{-1} \cdot g$. Moreover, we set $\mathcal{G}^{\omega}=r^{-1}(\{\omega\})$ for any unit $\omega \in \mathcal{G}^{0}$. One easily checks that $g \cdot h$ exists if and only if $r(h)=s(g)$.

The groupoids under consideration will always be measurable, i.e., they posses a $\sigma$-algebra $\mathcal{B}$ such that all relevant maps are measurable. More precisely, we require that $:: \mathcal{G}^{(2)} \rightarrow \mathcal{G},{ }^{-1}: \mathcal{G} \rightarrow \mathcal{G}, s, r: \mathcal{G} \rightarrow \mathcal{G}^{0}$ are measurable, where

$$
\mathcal{G}^{(2)}:=\left\{\left(g_{1}, g_{2}\right) \mid s\left(g_{1}\right)=r\left(g_{2}\right)\right\} \subset \mathcal{G}^{2}
$$

and $\mathcal{G}^{0} \subset \mathcal{G}$ are equipped with the induced $\sigma$-algebras. Analogously, $\mathcal{G}^{\omega} \subset \mathcal{G}$ are measurable spaces with the induced $\sigma$-algebras.

As mentioned above, the groupoid associated with (RSM) is simply $\mathcal{G}=\Omega \times \Gamma$ and the corresponding operations are defined as

$$
\begin{gathered}
(\omega, \gamma)^{-1}=\left(\gamma^{-1} \omega, \gamma^{-1}\right), \\
\left(\omega_{1}, \gamma_{1}\right) \cdot\left(\omega_{2}, \gamma_{2}\right)=\left(\omega_{1}, \gamma_{1} \gamma_{2}\right),
\end{gathered}
$$

where the left hand side of (41) is only defined if $\omega_{1}=\gamma_{1} \omega_{2}$. It is very useful to consider the elements $(\omega, \gamma)$ of this groupoid as the set of arrows $\gamma^{-1} \omega \stackrel{\gamma}{\longmapsto} \omega$. This yields a nice visualization of the operation $\cdot$ as concatenation of arrows and of the operation ${ }^{-1}$ as reversing the arrow. The units $\mathcal{G}^{0}=\{(\omega, \epsilon) \mid \omega \in \Omega\}$ can canonically be identified with the elements of the probability space $\Omega$. Via this identification, the maps $s$ and $r$ assign to each arrow its origin and its destination. Our groupoid can be seen as a bundle over the base space $\Omega$ of units with the fibers $\mathcal{G}^{\omega}=\{(\omega, \gamma) \mid \gamma \in \Gamma\} \cong \Gamma$. For simplicity, we henceforth refer to the set of units as $\Omega$ also in the setting of an abstract groupoid. The notions associated with the groupoid are illustrated in Figure 1 for both the abstract case and the concrete case of (RSM).

Next, we introduce an appropriate abstract object which corresponds to the Riemannian manifold $X$ in (RSM).

Definition 2.2. Let $\mathcal{G}$ be a measurable groupoid with the previously introduced notations. A triple $(\mathcal{X}, \pi, J)$ is called a (measurable) $\mathcal{G}$-space if the following properties are satisfied: $\mathcal{X}$ is a measurable space with associated $\sigma$-algebra $\mathcal{B}_{\mathcal{X}}$. The map $\pi: \mathcal{X} \rightarrow \Omega$ is measurable. Moreover, with $\mathcal{X}^{\omega}=\pi^{-1}(\{\omega\})$, the map $J$ assigns, to every $g \in \mathcal{G}$, an isomorphism $J(g): \mathcal{X}^{s(g)} \rightarrow \mathcal{X}^{r(g)}$ of measurable spaces with the properties $J\left(g^{-1}\right)=J(g)^{-1}$ and $J\left(g_{1} \cdot g_{2}\right)=J\left(g_{1}\right) \circ J\left(g_{2}\right)$ if $s\left(g_{1}\right)=r\left(g_{2}\right)$.

Note that a picture similar to Figure 1 exists for a $\mathcal{G}$-space $\mathcal{X}$.

An easy observation is that every groupoid $\mathcal{G}$ itself is a $\mathcal{G}$-space with $\pi=r$ and $J(g) h=g \cdot h$. 
The $\mathcal{G}$-space in (RSM) is given by $\mathcal{X}=\Omega \times X$ together with the maps $\pi(\omega, x)=\omega$ and

$$
J(\omega, \gamma): \mathcal{X}^{\gamma^{-1} \omega} \rightarrow \mathcal{X}^{\omega}, \quad J(\omega, \gamma)\left(\gamma^{-1} \omega, x\right)=(\omega, \gamma x) .
$$

Similarly to the groupoid $\mathcal{G}$, an arbitrary $\mathcal{G}$-space can be viewed as a bundle over the base $\Omega$ with fibers $\mathcal{X}^{\omega}$.

Our next aim is to exhibit natural measures on these objects. We first introduce families of measures on the fibers $\mathcal{G}^{\omega}$. In the case of (RSM), this can be viewed as an appropriate generalization of the Haar measure on $\Gamma$.

Definition 2.3. Let $\mathcal{G}$ be a measurable groupoid and the notation be given as above.

(a) A kernel of $\mathcal{G}$ is a map $\nu: \Omega \rightarrow \mathcal{M}(\mathcal{G})$ with the following properties:

- the map $\omega \mapsto \nu^{\omega}(f)$ is measurable for every $f \in \mathcal{F}^{+}(\mathcal{G})$,

- $\nu^{\omega}$ is supported on $\mathcal{G}^{\omega}$, i.e., $\nu^{\omega}\left(\mathcal{G}-\mathcal{G}^{\omega}\right)=0$.

(b) A transverse function $\nu$ of $\mathcal{G}$ is a kernel satisfying the following invariance condition

$$
\int_{\mathcal{G}^{s(g)}} f(g \cdot h) d \nu^{s(g)}(h)=\int_{\mathcal{G}^{r(g)}} f(k) d \nu^{r(g)}(k)
$$

for all $g \in \mathcal{G}$ and $f \in \mathcal{F}^{+}\left(\mathcal{G}^{r(g)}\right)$.

In (RSM) the discreteness of $\Gamma$ implies that any kernel $\nu$ can be identified with a function $L \in \mathcal{F}^{+}(\Omega \times \Gamma)$ via $\nu^{\omega}=\sum_{\gamma \in \Gamma} L(\omega, \gamma) \delta_{(\omega, \gamma)}$. For an arbitrary unimodular group $\Gamma$, the Haar measure $m_{\Gamma}$ induces a transverse function $\nu$ by $\nu^{\omega}=m_{\Gamma}$ for all $\omega \in \Omega$ on the groupoid $\Omega \times \Gamma$ via the identification $\mathcal{G}^{\omega} \cong \Gamma$.

In the next definition we introduce appropriate measures on the base space $\Omega$ of an abstract groupoid $\mathcal{G}$.

Definition 2.4. Let $\mathcal{G}$ be a measurable groupoid with a transverse function $\nu$. A measure $\mu$ on the base space $\left(\Omega, \mathcal{B}_{\Omega}\right)$ of units is called $\nu$-invariant (or simply invariant, if there is no ambiguity in the choice of $\nu$ ) if

$$
\mu \circ \nu=(\mu \circ \nu)^{\sim},
$$

where $(\mu \circ \nu)(f)=\int_{\Omega} \nu^{\omega}(f) d \mu(\omega)$ and $(\mu \circ \nu)^{\sim}(f)=(\mu \circ \nu)(\tilde{f})$ with $\tilde{f}(g)=$ $f\left(g^{-1}\right)$.

In (RSM) it can easily be checked that, with the above choice $\nu \equiv m_{\Gamma}$, a measure $\mu$ on $\left(\Omega, \mathcal{B}_{\Omega}\right)$ is $\nu$-invariant if and only if $\mu$ is $\Gamma$-invariant in the classical sense, see [Con79, Cor. II.7] as well. Thus a canonical choice for a $m_{\Gamma}$-invariant measure on $\Omega$ is $\mathbb{P}$.

Analogously to transverse functions on the groupoid, we introduce a corresponding fiberwise consistent family $\alpha$ of measures on the $\mathcal{G}$-space, see the next definition. We refer to the resulting object $(\mathcal{X}, \alpha)$ as a random variable in the sense of Connes. These random variables are useful substitutes for measurable functions on the quotient space $\Omega / \Gamma$ with values in $X$. Measurable functions on $\Omega / \Gamma$ can be identified with $\Gamma$-invariant measurable functions on $\Omega$. Note that, 


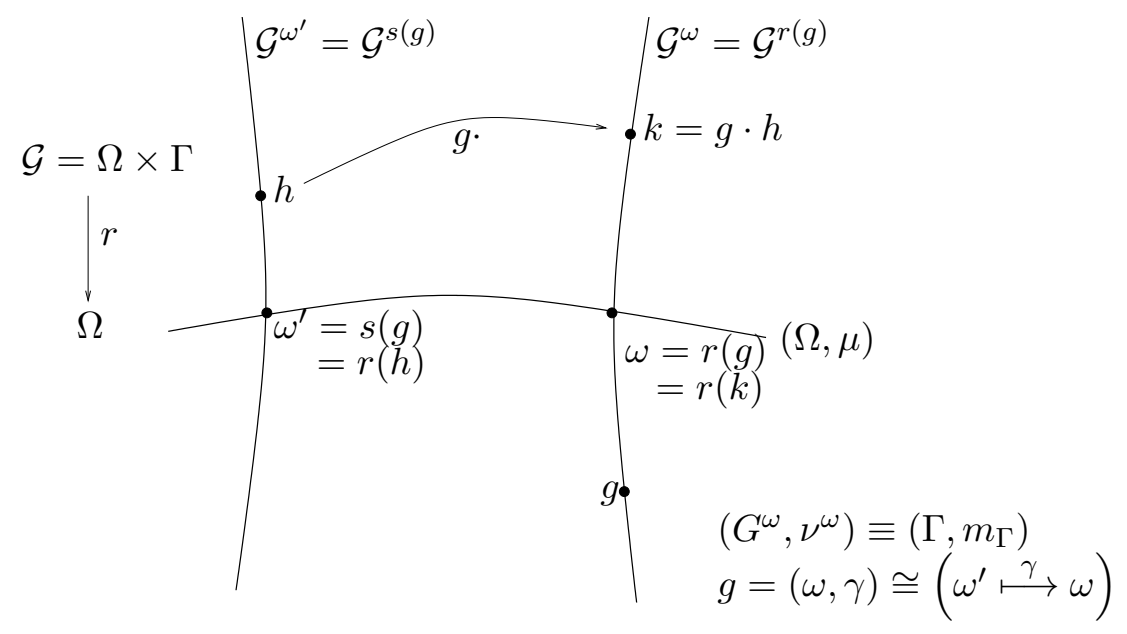

FiguRE 1. Notations of the groupoid $\mathcal{G}=\Omega \times \Gamma$ in $(\mathrm{RSM})$

in the case of an ergodic action of $\Gamma$ on $\Omega$, there are no nontrivial $\Gamma$-invariant measurable functions, whereas there are usually lots of random variables in the sense of Connes (see below for examples).

Definition 2.5. Let $\mathcal{G}$ be a measurable groupoid and $\mathcal{X}$ be a $\mathcal{G}$-space. A choice of measures $\alpha: \Omega \rightarrow \mathcal{M}(\mathcal{X})$ is called a random variable (in the sense of Connes) with values in $\mathcal{X}$ if it has the following properties

- the map $\omega \mapsto \alpha^{\omega}(f)$ is measurable for every $f \in \mathcal{F}^{+}(\mathcal{X})$,

- $\alpha^{\omega}$ is supported on $\mathcal{X}^{\omega}$, i.e., $\alpha^{\omega}\left(\mathcal{X}-\mathcal{X}^{\omega}\right)=0$,

- $\alpha$ satisfies the following invariance condition

$$
\int_{\mathcal{X}^{s(g)}} f(J(g) p) d \alpha^{s(g)}(p)=\int_{\mathcal{X}^{r(g)}} f(q) d \alpha^{r(g)}(q)
$$

for all $g \in \mathcal{G}$ and $f \in \mathcal{F}^{+}\left(\mathcal{X}^{r(g)}\right)$.

To simplify notation, we write $g h$ respectively $g p$ for $g \cdot h$ respectively $J(g) p$.

The general setting for the sequel consists of a groupoid $\mathcal{G}$ equipped with a fixed transverse function $\nu$ and an $\nu$-invariant measure $\mu$ on $\Omega$, and a fixed random variable $(\mathcal{X}, \alpha)$. We use the follwing notation for the "averaging" of a $u \in \mathcal{F}^{+}(\mathcal{X})$ with respcet to $\nu$

$$
\nu * u_{0}(p):=\int_{\mathcal{G}^{\pi(p)}} u_{0}\left(g^{-1} p\right) d \nu^{\pi(p)}(g) \quad \text { for } p \in \mathcal{X} .
$$

We will need the following further assumptions in order to apply the integration theory developed in Con79.

Definition 2.6. Let $(\mathcal{G}, \nu, \mu)$ be a measurable groupoid and $(\mathcal{X}, \alpha)$ be a random variable on the associated $\mathcal{G}$-space $\mathcal{X}$ satisfying the following two conditions

(6) The $\sigma$-algebras $\mathcal{B}_{\mathcal{X}}$ and $\mathcal{B}_{\Omega}$ are generated by a countable family of sets, all of which have finite measure, w.r.t. $\mu \circ \alpha$ (respectively w.r.t. $\mu$ ). 
(7) There exists a strictly positive function $u_{0} \in \mathcal{F}^{+}(\mathcal{X})$ satisfying $\nu * u_{0}(p)=1$ for all $p \in \mathcal{X}$.

Then we call the tupel $\left(\mathcal{G}, \nu, \mu, \mathcal{X}, \alpha, u_{0}\right)$ an admissible setting.

Before continuing our investigation let us shortly discuss the relevance of the above conditions: Condition (6) is a strong type of separability condition for the Hilbert space $L^{2}(\mathcal{X}, \mu \circ \alpha)$. It enables us to use the techniques from direct integral theory discussed in Appendix A which are crucial to the considerations in Section 3 ,

Condition (7) is important to apply Connes' non-commutative integration theory. Namely, it says that $(\mathcal{X}, J)$ is proper in the sense of Lemma III.2 and Definition III.3 of [Con79]. Therefore $(\mathcal{X}, J)$ is square integrable by Proposition IV.12 of [Con79]. This square integrability in turn is a key condition for the applications of Con79] we give in Sections 3 to 5 .

On an intuitive level, (7) can be understood as providing an "embedding" of $\mathcal{G}$ into $\mathcal{X}$. Namely, every $u \in \mathcal{F}(\mathcal{X})$ with $\nu * u \equiv 1$ gives rise to the fibrewise defined map $q=q_{u}: \mathcal{F}(\mathcal{G}) \rightarrow \mathcal{F}(\mathcal{X})$ by

$$
q(f)(p):=\int_{\mathcal{G}^{\pi(p)}} u\left(g^{-1} p\right) f(g) d \nu^{\pi(p)}(g)
$$

for all $p \in \mathcal{X}$. Note that the convolution property of $u$ implies that the map (8) satisfies $q\left(1_{\mathcal{G}}\right)=1_{\mathcal{X}}$. Moreover, $q$ can be used to obtain new functions $w \in \mathcal{F}(\mathcal{X})$ satisfying $\nu * w \equiv 1$. This is the statement of the next proposition.

Proposition 2.7. Let $u \in \mathcal{F}^{+}(\mathcal{X})$ with $\nu * u \equiv 1$ be given, and $q$ be as above. For any function $f \in \mathcal{F}(\mathcal{G})$ with $\nu(\tilde{f}) \equiv 1$ on $\Omega$ we have $\nu * q(f) \equiv 1$.

Proof. The proof is given by the following direct calculation with $\omega=\pi(p) \in \Omega$ :

$$
\begin{aligned}
(\nu * q(f))(p) & =\int_{\mathcal{G}^{\omega}} q(f)\left(g^{-1} p\right) d \nu^{\omega}(g) \\
& =\int_{\mathcal{G}^{\omega}} \int_{\mathcal{G}^{s(g)}} u\left(h^{-1} g^{-1} p\right) f(h) d \nu^{s(g)}(h) d \nu^{\omega}(g) \\
(\nu \text { transverse function }) & =\int_{\mathcal{G}^{\omega}} \int_{\mathcal{G}^{\omega}} u\left(k^{-1} p\right) f\left(g^{-1} k\right) d \nu^{\omega}(k) d \nu^{\omega}(g) \\
\text { (Fubini) }) & =\int_{\mathcal{G}^{\omega}} u\left(k^{-1} p\right) \int_{\mathcal{G}^{\omega}} f\left(g^{-1} k\right) d \nu^{\omega}(g) d \nu^{\omega}(k) \\
& =\int_{\mathcal{G}^{\omega}} u\left(k^{-1} p\right) \int_{\mathcal{G}^{\omega}} \tilde{f}\left(k^{-1} g\right) d \nu^{\omega}(g) d \nu^{\omega}(k) \\
(\nu(\tilde{f}) \equiv 1) & =\int_{\mathcal{G}^{\omega}} u\left(k^{-1} p\right) d \nu^{\omega}(k) \\
& =1 .
\end{aligned}
$$

Note that in the above calculation the integration variable $g$ has the property $r(g)=\pi(p)=\omega$. This finishes the proof. 
Remark 2.8. We consider examples of admissible settings in the case (RSM). Recall that we will identify $\mathcal{G}^{\omega}$ with $\Gamma$ and $\mathcal{X}^{\omega}$ with $X$ for all $\omega \in \Omega$. A transverse function of $\mathcal{G}$ is given by copies of the Haar measure: $\nu^{\omega}=m_{\Gamma}$ for all $\omega \in \Omega$. $\mathcal{X}$ together with the Riemannian volume forms $\lambda^{\omega}$ (corresponding to the metrics $g_{\omega}$ ) on the fibers $\mathcal{X}^{\omega} \equiv X$ is an example of a random variable. This will be shown next by discussing validity of conditions (7) and (6).

Condition (7): Let $\mathcal{D}$ be a fundamental domain for the $\Gamma$ action on $X$ such that $\bigcup_{\gamma \in \Gamma} \gamma \mathcal{D}=X$ is a disjoint union, c.f. [Ra91, §6.5]. Then every function $v \in \mathcal{F}(\Gamma)$ with $\sum_{\gamma \in \Gamma} v(\gamma)=1$ gives rise to a $u_{0}$ satisfying $\nu * u_{0} \equiv 1$ by

$$
u_{0}(\omega, x)=v(\gamma)
$$

where $\gamma \in \Gamma$ is the unique element with $x \in \gamma \mathcal{D}$. Note that this construction assigns to a strictly positive $v$, again a strictly positive $u_{0}$ on $\mathcal{X}$. Hence the setting $\left(\Omega \times \Gamma, m_{\Gamma}, \mathbb{P}, \Omega \times X, \lambda, u_{0}\right)$ satisfies condition (7).

Condition (6): This condition is clearly satisfied if the $\sigma$-algebra of $\Omega$ is countably generated. In the case (RSM) (I) this countability condition can always be achieved by passing to an equivalent version of the defining stochastic process. Namely, given a random potential $V: \Omega \times X \rightarrow \mathbb{R}$, we construct a stochastic process $\tilde{V}: \tilde{\Omega} \times X \rightarrow \mathbb{R}$ with the same finite dimensional distributions such that (6) is satisfied, c.f. e.g. [GS79, PF92].

Assume at first that the random potential $V: \Omega \times X \rightarrow \mathbb{R}$ can be written as

$$
V_{\omega}(x)=\sum_{\gamma \in \Gamma} f_{\gamma}\left(\omega, \gamma^{-1} x\right) .
$$

Here $\left(f_{\gamma}\right)_{\gamma \in \Gamma}$ is a sequence of measurable functions on the probability space $\Omega$ with values in a separable Banach space $B$ of functions on $X$. Such models have been studied by Kirsch in [Kir81]. Note that the Borel- $\sigma$-algebra $\mathcal{B}_{B}$ is generated by a countable set $\mathcal{E} \subset \mathcal{B}_{B}$. In this case $\Omega$ can be chosen to be the canonical probability space $B^{\Gamma}$. Its $\sigma$-algebra is generated by a countable family of cylinder sets of the form

$$
\left\{\omega \in B^{\Gamma} \mid \forall \gamma \in H^{n}: \omega(\gamma) \in M_{\gamma}\right\}
$$

where $H$ denotes a finite set of generators of $\Gamma, n \in \mathbb{N}$ and $M_{\gamma} \in \mathcal{E}$ for each $\gamma$. An appropriate choice for the (separable) Banach space $B$ is:

$$
\ell^{1}\left(L^{p}\right)(X):=\left\{f: X \rightarrow \mathbb{C} \mid \int_{\Gamma}\left(\int_{\gamma \mathcal{D}}|f(x)|^{p} d \lambda(x)\right)^{1 / p} d m_{\Gamma}(\gamma)<\infty\right\}
$$

or

$$
L^{p}\left(\ell^{1}\right)(X):=\left\{f: X \rightarrow \mathbb{C} \mid\left(\int_{\mathcal{D}}\left(\int_{\Gamma}\left|f\left(\gamma^{-1} x\right)\right| d m_{\Gamma}(\gamma)\right)^{p} d \lambda(x)\right)^{1 / p}<\infty\right\} .
$$

Here $\mathcal{D}$ denotes an arbitrary $\Gamma$-fundamental domain in $X$. We have the inclusion

$$
L^{p}(X) \subset \ell^{1}\left(L^{p}(X)\right) \subset L^{p}\left(\ell^{1}(X)\right)
$$


and $L^{p}\left(\ell^{1}(X)\right)$ is separable, cf. [Kir81, Kir89]. More correctly, one could use the notation $\ell^{1}\left(\Gamma, L^{p}(\mathcal{D})\right)$ for $\ell^{1}\left(L^{p}\right)(X)$ and $L^{p}\left(\mathcal{D}, \ell^{1}(\Gamma)\right)$ for $L^{p}\left(\ell^{1}\right)(X)$.

Now we show that actually all models which are of the the type (RSM) (I) can be written in the way (91). Let $u: X \rightarrow \mathbb{R}^{+}$be a bounded measurable function such that $\sum_{\gamma \in \Gamma} u\left(\gamma^{-1} x\right)=1$ for all $x \in X$. Let $\phi=V u$. Then

$V(\omega, x)=\sum_{\gamma \in \Gamma} V(\omega, x) u\left(\gamma^{-1} x\right)=\sum_{\gamma \in \Gamma} V\left(\gamma^{-1} \omega, \gamma^{-1} x\right) u\left(\gamma^{-1} x\right)=\sum_{\gamma \in \Gamma} \phi\left(\gamma^{-1} \omega, \gamma^{-1} x\right)$.

Setting $f_{\gamma}(\omega, x)=\phi\left(\gamma^{-1} \omega, x\right)$ we have a representation as in (9).

The regularity assumptions on $V$ in $(\mathrm{RSM})$ imply that $V(\omega, \cdot) \in L_{\text {loc,unif }}^{p}(X)$ for all $\omega \in \Omega$ and all $p \in[1, \infty]$. Here $L_{\text {loc,unif }}^{p}(X)=\ell^{\infty}\left(L^{p}\right)(X)$ denotes the set of locally $L^{p}$-integrable functions $f$ such that the $L^{p}$-norm of $f \chi_{\gamma \mathcal{D}}$ is uniformly bounded in $\gamma \in \Gamma$. The choice $u=\chi_{\mathcal{D}}$ yields $\phi(\omega, \cdot) \in L^{p}(X)$. Thus the functions $f_{\gamma}(\omega, \cdot)$ are in the Banach space $B=L^{p}(X)$.

Summarizing the above considerations, we conclude that (RSM) (I) satisfies both Condition (6) and (7) (after a suitable modification of the underlying probability space). The case (RSM) (II) can be treted similarly.

A crucial fact about the integration of random variables is given in the following lemma, essentially contained in (the proof of) Lemma III.1 in [Con79]. We include a proof for the convenience of the reader.

Lemma 2.9. Let $\mathcal{G}$ be a groupoid with transverse function $\nu$ and $\nu$-invariant measure $\mu$. Let furthermore $\mathcal{X}$ be a $\mathcal{G}$-space.

(a) For a given transverse function $\phi$ on $\mathcal{G}$, the integral $\int_{\Omega} \phi^{\omega}(f) d \mu(\omega)$ does not depend on $f \in \mathcal{F}^{+}(\mathcal{G})$, provided $f$ satisfies $\nu(\tilde{f}) \equiv 1$.

(b) For a given random variable $\alpha$ with values in $\mathcal{X}$ the integral $\int_{\Omega} \alpha^{\omega}(u) d \mu(\omega)$ does not depend on $u$, provided $u \in \mathcal{F}^{+}(\mathcal{X})$ satisfies $\nu * u \equiv 1$.

Proof. We prove first part (b). So let $(\mathcal{X}, \alpha)$ and $u, u^{\prime} \in \mathcal{F}(\mathcal{X})$ with $\nu * u^{\prime} \equiv$ $\nu * u \equiv 1$ be given. Inserting $1 \equiv \nu * u^{\prime}$, we calculate

$$
\begin{aligned}
\int_{\Omega} \alpha^{\omega}(u) d \mu(\omega) & =\int_{\Omega} \int_{\mathcal{X}^{\omega}} u(p) \cdot 1 \cdot d \alpha^{\omega}(p) d \mu(\omega) \\
\text { (Fubini) } & =\int_{\Omega} \int_{\mathcal{G}^{\omega}} \int_{\mathcal{X}^{\omega}} u^{\prime}\left(g^{-1} p\right) u(p) d \alpha^{\omega}(p) d \nu^{\omega}(g) d \mu(\omega) \\
(\mu \text { inv. }) & =\int_{\Omega} \int_{\mathcal{G}^{\omega}} \int_{\mathcal{X}^{s(g)}} u^{\prime}\left(g p^{\prime}\right) u\left(p^{\prime}\right) d \alpha^{s(g)}\left(p^{\prime}\right) d \nu^{\omega}(g) d \mu(\omega) \\
(\alpha \text { inv. }) & =\int_{\Omega} \int_{\mathcal{G}^{\omega}} \int_{\mathcal{X}^{\omega}} u^{\prime}(p) u\left(g^{-1} p\right) d \alpha^{\omega}(p) d \nu^{\omega}(g) d \mu(\omega) .
\end{aligned}
$$

Another application of Fubini and use of $\nu * u \equiv 1$ then gives $\int_{\Omega} \alpha^{\omega}(u) d \mu(\omega)=$ $\int_{\Omega} \alpha^{\omega}\left(u^{\prime}\right) d \mu(\omega)$ and the proof of (b) is finished.

Note that every groupoid $\mathcal{G}$ is a $\mathcal{G}$-space $\mathcal{X}$. Furthermore, in this case the convolution condition $\nu * u \equiv 1$ translates into $\nu(\tilde{f}) \equiv 1$. This proves (a). 
Finally, we introduce, for a given $\mathcal{G}$-space $\mathcal{X}$, a new $\mathcal{G}$-space $\mathcal{X} \times_{\Omega} \mathcal{X}$. It consists of the set

$$
\mathcal{X} \times \Omega \mathcal{X}:=\{(p, q) \mid \pi(p)=\pi(q)\} \subset \mathcal{X} \times \mathcal{X} .
$$

equipped with the induced $\sigma$-algebra. The corresponding maps $\pi$ and $J$ of $\mathcal{X} \times_{\Omega} \mathcal{X}$ are defined in an obvious way. If $\mathcal{X}$ is actually a random variable with measures $\left(\alpha^{\omega}\right)_{\omega}$, then $\mathcal{X} \times_{\Omega} \mathcal{X}$ inherits the structure of a random variable by setting

$$
\left(\alpha \times_{\Omega} \alpha\right)^{\omega}:=\alpha^{\omega} \otimes \alpha^{\omega} .
$$

\section{The von Neumann Algebra $\mathcal{N}(\mathcal{G}, \mathcal{X})$}

In this section we discuss how a von Neumann Algebra arises naturally for an admissible setting $\left(\mathcal{G}, \nu, \mu, \mathcal{X}, \alpha, u_{0}\right)$, cf. Con79. The random operators we are interested in are affiliated to this von Neumann algebra. Recall that a selfadjoint operator is called affiliated to a von Neumann algebra if its spectral family is contained in the von Neumann algebra.

A $\mathcal{G}$-space $\mathcal{X}$ is, by definition, a bundle over $\Omega=\mathcal{G}^{(0)}$ via $\pi: \mathcal{X} \longrightarrow \Omega$. This bundle structure of a random variable $(\mathcal{X}, \alpha)$ induces a bundle structure of $L^{2}(\mathcal{X}, \mu \circ \alpha)$ : Using the Fubini Theorem, we can associate with $f \in L^{2}(\mathcal{X}, \mu \circ \alpha)$ a family $\left(f_{\omega}\right)_{\omega \in \Omega}$ of functions

$$
f_{\omega} \in L^{2}\left(\mathcal{X}^{\omega}, \alpha^{\omega}\right) \text { such that } f(x)=f_{\pi(x)}(x)
$$

for $\mu$-almost all $\omega \in \Omega$. This way of decomposing will be a key tool in the sequel. On the technical level, this is very conveniently expressed using direct integral theory (see e.g. [Dix81]) and the fact that there is a canonical isomorphism

$$
L^{2}(\mathcal{X}, \mu \circ \alpha) \simeq \int_{\Omega}^{\oplus} L^{2}\left(\mathcal{X}^{\omega}, \alpha^{\omega}\right) d \mu(\omega) .
$$

As direct integral theory tends to be rather technical, we try to avoid it in the sequel and rather give direct arguments which, however, are inspired by the general theory (cf. Appendix A).

A special role will be played by those operators which respect the bundle structure of $L^{2}(\mathcal{X}, \mu \circ \alpha)$ given by (10). Namely, we say that the (not necessarily bounded) operator $A: L^{2}(\mathcal{X}, \mu \circ \alpha) \longrightarrow L^{2}(\mathcal{X}, \mu \circ \alpha)$ is decomposable if there exist operators $A_{\omega}: L^{2}\left(\mathcal{X}^{\omega}, \alpha^{\omega}\right) \longrightarrow L^{2}\left(\mathcal{X}^{\omega}, \alpha^{\omega}\right)$ such that $(A f)(x)=\left(A_{\omega} f_{\omega}\right)(x)$, for almost every $\omega \in \Omega$. We then write $A=\int_{\Omega}^{\oplus} A_{\omega} d \mu(\omega)$.

Let the groupoid $(\mathcal{G}, \mu, \nu)$ and the random variable $(\mathcal{X}, \alpha)$ be as in the last section. For $g \in \mathcal{G}$, let the unitary operator $U_{g}$ be given by

$$
U_{g}: L^{2}\left(\mathcal{X}^{s(g)}, \alpha^{s(g)}\right) \longrightarrow L^{2}\left(\mathcal{X}^{r(g)}, \alpha^{r(g)}\right), U_{g} f(p):=f\left(g^{-1} p\right) .
$$

A family $\left(A_{\omega}\right)_{\omega \in \Omega}$ of bounded operators $A_{\omega}: L^{2}\left(\mathcal{X}^{\omega}, \alpha^{\omega}\right) \rightarrow L^{2}\left(\mathcal{X}^{\omega}, \alpha^{\omega}\right)$ is called a bounded random operator if it satisfies :

- $\omega \mapsto\left\langle g_{\omega}, A_{\omega} f_{\omega}\right\rangle$ is measurable for arbitrary $f, g \in L^{2}(\mathcal{X}, \mu \circ \alpha)$.

- There exists a $C \geq 0$ with $\left\|A_{\omega}\right\| \leq C$ for almost all $\omega \in \Omega$. 
- The equivariance condition $A_{r(g)}=U_{g} A_{s(g)} U_{g}^{*}$ for all $g \in \mathcal{G}$ is satisfied. Two bounded random operators $\left(A_{\omega}\right),\left(B_{\omega}\right)$ are called equivalent, $\left(A_{\omega}\right) \sim\left(B_{\omega}\right)$ if $A_{\omega}=B_{\omega}$ for $\mu$-almost every $\omega \in \Omega$. Each equivalence class of bounded random operators $\left(A_{\omega}\right)$ gives rise to a bounded operator $A$ on $L^{2}(\mathcal{X}, \mu \circ \alpha)$ by $A f(p):=A_{\pi(p)} f_{\pi(p)}$ (cf. Appendix A). This allows us to identify the class of $\left(A_{\omega}\right)$ with the bounded operator $A$. The following is the main theorem on the structure of the space of random operators.

Theorem 3.1. The set $\mathcal{N}(\mathcal{G}, \mathcal{X})$ of classes of bounded random operators is a von Neumann algebra.

Proof. This follows immediately from [Con79, Thm. V.2].

To get some insight we give a proof of Theorem 3.1 for the particular case of (RSM). In this example we can canonically identify $L^{2}\left(\mathcal{X}^{\omega}, \alpha^{\omega}\right)$ with $L^{2}\left(X, \lambda^{\omega}\right)$ and $L^{2}(\mathcal{X}, \mu \circ \alpha)$ with $L^{2}(\Omega \times X, \mathbb{P} \circ \lambda)$. Under this identification a bounded random operator is just a family of uniformly bounded operators

$$
A_{\omega}: L^{2}\left(X, \lambda^{\omega}\right) \rightarrow L^{2}\left(X, \lambda^{\omega}\right), \omega \in \Omega
$$

with $(\omega, x) \mapsto\left(A_{\omega} f_{\omega}\right)(x)$ measurable and $\omega \mapsto A_{\omega} f_{\omega} \in L^{2}\left(X, \lambda^{\omega}\right)$ for every $f \in L^{2}(\Omega \times X, \mathbb{P} \circ \lambda)$, satisfying the equivariance condition

$$
A_{\omega}=U_{(\omega, \gamma)} A_{\gamma^{-1} \omega} U_{(\omega, \gamma)}^{*},
$$

where we write $U_{g}$ as $U_{(\omega, \gamma)}$ due to the product structure of $\mathcal{G}=\Omega \times \Gamma$.

In this case we can also associate with $\left(A_{\omega}\right)$ an operator $A$ on the Hilbert space $L^{2}(\Omega \times X, \mathbb{P} \circ \lambda)$ by setting $(A f)(\omega, x) \equiv\left(A_{\omega} f_{\omega}\right)(x)$. Now, (12) implies

$$
A \widetilde{U}_{\gamma}=\widetilde{U}_{\gamma} A \text {, }
$$

where $\widetilde{U}_{\gamma}$ is the unitary operator on $L^{2}(\Omega \times X, \mathbb{P} \circ \lambda)$ defined by

$$
\left(\widetilde{U}_{\gamma} f\right)_{\omega}(x)=f_{\gamma^{-1} \omega}\left(\gamma^{-1} x\right) .
$$

Conversely, one can show for a decomposable operator $A$ that (13) implies (12) $\mathbb{P}$-almost everywhere. Now, a suitable averaging procedure and a change of the fibers $A_{\omega}$ on a set of $\mathbb{P}$-measure zero yields (12) for all $\omega$ (cf. [Len99. or Con79, p. 88]).

It is well known that a bounded operator $A$ on $L^{2}(\Omega \times X)$ is decomposable, i.e. $A=\int_{\Omega}^{\oplus} A_{\omega} d \mathbb{P}(\omega)$ for a suitable family $\left(A_{\omega}\right)$, if and only if $A$ commutes with the multiplication operators $M_{h \circ \pi}$ for every $h \in L^{\infty}(\Omega, \mathbb{P})$ (see, e.g., Dix81, Thm. 1 in II.2.5]). Summarizing these considerations, we conclude

$$
\mathcal{N}(\Omega \times \Gamma, \Omega \times X)=\left\{\widetilde{U}_{\gamma} \mid \gamma \in \Gamma\right\}^{\prime} \cap\left\{M_{h \circ \pi} \mid h \in L^{\infty}(\Omega, \mathbb{P})\right\}^{\prime},
$$

where $S^{\prime}$ denotes the commutant of a set $S$ of operators. Obviously, $\left\{\widetilde{U}_{\gamma} \mid \gamma \in\right.$ $\Gamma\}$ and $\left\{M_{h \circ \pi} \mid h \in L^{\infty}(\Omega, \mathbb{P})\right\}$ are closed under taking adjoints. Thus, their commutants are von Neumann algebras. This reasoning shows directly, in the case of (RSM), that the space of random operators is a von Neumann algebra. 


\section{The canonical trace on $\mathcal{N}(\mathcal{G}, \mathcal{X})$}

In this section we start with an admissible setting $\left(\mathcal{G}, \nu, \mu, \mathcal{X}, \alpha, u_{0}\right)$ and its associated von Neumann algebra $\mathcal{N}(\mathcal{G}, \mathcal{X})$ of bounded operators on $L^{2}(\mathcal{X}, \mu \circ \alpha)$. Let $\mathcal{N}^{+}(\mathcal{G}, \mathcal{X})$ denote the set of non negative selfadjoint operators in $\mathcal{N}(\mathcal{G}, \mathcal{X})$. We will show that every operator $A \in \mathcal{N}^{+}(\mathcal{G}, \mathcal{X})$ gives rise to a new random variable $\left(\mathcal{X}, \beta_{A}\right)$. Integrating this random variable, we obtain a weight on $\mathcal{N}(\mathcal{G}, \mathcal{X})$. Under certain (mild) assumptions this weight can be shown to be a trace.

We start by associating a transversal function as well as a random variable with each element in $\mathcal{N}^{+}(\mathcal{G}, \mathcal{X})$. In the following, $q_{\omega}(f)$ denotes the restriction of $q(f)$ as defined in (8) to the fiber $\mathcal{X}^{\omega}$.

Lemma 4.1. Let $A \in \mathcal{N}^{+}(\mathcal{G}, \mathcal{X})$.

(a) Then $\phi_{A}$, given by $\phi_{A}^{\omega}(f):=\operatorname{tr}\left(A_{\omega} M_{q_{\omega}(f)}\right), f \in \mathcal{F}\left(\mathcal{G}^{\omega}\right)$, defines a transverse function.

(b) Then $\beta_{A}$, given by $\beta_{A}^{\omega}(f):=\operatorname{tr}\left(A_{\omega} M_{f}\right), f \in \mathcal{F}\left(\mathcal{X}^{\omega}\right)$, is a random variable.

Proof. This follows by direct calculation using the equivariance properties of the family $A_{\omega}$ and the $q_{\omega}$.

Let us recall the following definitions. A weight on a von Neumann algebra $\mathcal{N}$ is a map $\tau: \mathcal{N}^{+} \rightarrow[0, \infty]$ satisfying $\tau(A+B)=\tau(A)+\tau(B)$ and $\tau(\lambda A)=\lambda \tau(A)$ for arbitrary $A, B \in \mathcal{N}^{+}$and $\lambda \geq 0$. The weight is called normal if $\tau\left(A_{n}\right)$ converges to $\tau(A)$ whenever $A_{n}$ is an increasing sequence of operators (i.e. $\left.A_{n} \leq A_{n+1}, n \in \mathbb{N}\right)$ converging strongly to $A$. It is called faithful if $\tau(A)=0$ implies $A=0$. It is called semifinite if $\tau(A)=\sup \{\tau(B): B \leq A, \tau(B)<\infty\}$. If a weight $\tau$ satisfies $\tau\left(C C^{*}\right)=\tau\left(C^{*} C\right.$ ) for arbitrary $C \in \mathcal{N}$ (or equivalently $\tau\left(U A U^{*}\right)=\tau(A)$ for arbitrary $A \in \mathcal{N}^{+}$and unitary $U \in \mathcal{N}$, cf. [Dix81, Cor. 1 in I.6.1]), it is called a trace.

Theorem 4.2. For $A \in \mathcal{N}^{+}(\mathcal{G}, \mathcal{X})$, the expression

$$
\tau(A):=\int_{\Omega} \operatorname{tr}\left(A_{\omega}^{\frac{1}{2}} M_{u_{\omega}} A_{\omega}^{\frac{1}{2}}\right) d \mu(\omega)=\int_{\Omega} \operatorname{tr}\left(M_{u_{\omega}}^{\frac{1}{2}} A_{\omega} M_{u_{\omega}}^{\frac{1}{2}}\right) d \mu(\omega)
$$

does not depend on $u \in \mathcal{F}^{+}(\mathcal{X})$ provided $\nu * u \equiv 1$.

(a) The map $\tau: \mathcal{N}^{+}(\mathcal{G}, \mathcal{X}) \longrightarrow[0, \infty]$ is a faithful, semifinite normal weight on $\mathcal{N}(\mathcal{G}, \mathcal{X})$.

(b) If the groupoid $\mathcal{G}$ satisfies the freeness condition

$$
r^{-1}(\omega) \cap s^{-1}(\omega)=\{\omega\} \text { for } \mu \text {-almost all } \omega \in \Omega,
$$

then $\tau$ is a trace.

Proof. That $\tau$ is independent of $u$ follows easily from Lemma 2.9 and Lemma 4.1.

(a) The proof that $\tau$ is a normal weight is straightforward. The proof of the semifiniteness of $\tau$ is not simple and we refer the reader to Theorem VI.2 in Con79. To show that $\tau$ is faithful, assume $\tau(A)=0$ for $A \in \mathcal{N}^{+}(\mathcal{G}, \mathcal{X})$. This 
implies $\operatorname{tr}\left(M_{u_{\omega}} A_{\omega}\right)=0$ for almost every $\omega$. As the trace $\operatorname{tr}$ is faithful, this implies $M_{u_{\omega}} A_{\omega}=0$ for almost every $\omega$. Choosing a strictly positive function $u$ (e.g. $u=u_{0}$ ), we infer $A_{\omega}=0$ for almost every $\omega$ and we see that $\tau$ is faithful. (b) The freeness condition (14) easily shows that Corollary VI.7 of [Con79] is applicable and the statement follows.

Remark 4.3. (i) Property (14) can be slightly relaxed (cf. Con79]). However, some condition of this form is necessary to prove the trace property in the generality addressed in the theorem. It will turn out to be dispensable in many concrete cases. Indeed, we will see below that $\tau$ is a trace in the case of (RSM) without any condition of this form. However, when considering factorial type properties this condition again plays an important role.

(ii) The motivation to call property (14) a freeness condition is easily understood in the context of (RSM). Namely, in this case (14) is equivalent to

$$
\mathbb{P}\left(\omega \mid \gamma^{-1} \omega=\omega\right)=0 \text { for all } \gamma \in \Gamma \backslash\{\epsilon\} .
$$

(iii) Given condition (14) and some ergodicity assumptions (see the next section), it follows from [Con79] that the von Neumann algebra is actually a factor. In this case the trace $\tau$ is unique (up to a scalar factor).

We are now heading towards an alternative direct proof of part (b) of Theorem 4.2 in the particular case (RSM). This proof will be based on a study of $\tau$ for certain operators with a kernel. It will in fact be more general, in that it does not use the freeness condition (14) on the action of $\Gamma$ on $\Omega$. Along our way, we will also find an instructive formula for $\tau$ on these operators. Let us emphasize that the approach to prove that $\tau$ is a trace given below is in no way restricted to (RSM) (cf. [LS01, LS04] for related material).

An operator $K$ on $L^{2}(\mathcal{X}, \mu \circ \alpha)$ is called a Carleman operator (cf. Wei80] for further details) if there exists a $k \in \mathcal{F}\left(\mathcal{X} \times_{\Omega} \mathcal{X}\right)$ with

$$
k(p, \cdot) \in L^{2}\left(\mathcal{X}^{\pi(p)}, \alpha^{\pi(p)}\right) \text { for all } p \in \mathcal{X}
$$

such that

$$
K f(p)=\int_{\mathcal{X}^{\pi(p)}} k(p, q) f(q) d \alpha^{\pi(p)}(q)=: K_{\pi(p)} f_{\pi(p)}(p) .
$$

This $k$ is called the kernel of $K$ : Obviously, $K=\int_{\Omega}^{\oplus} K_{\omega} d \mu$. Let $\mathcal{K}$ be the set of all Carleman operators satisfying for all $g \in \mathcal{G}$

$$
k(g p, g q)=k(p, q) \text { for } \alpha^{\pi(g)} \times \alpha^{\pi(g)} \text { almost all } p, q .
$$

Proposition 4.4. $\mathcal{K}$ is a right ideal in $\mathcal{N}(\mathcal{G}, \mathcal{X})$.

Proof. Obviously, $K \in \mathcal{K}$ is decomposable. Thus, to show that $\mathcal{K}$ is a subset of $\mathcal{N}(\mathcal{G}, \mathcal{X})$, it suffices to show the equivariance condition. But this is immediate from (16). Thus, it remains to show that $\mathcal{K}$ is a right ideal. This follows from a standard calculation in the theory of Carleman operators:

$$
K A f(p)=K_{\pi(p)} A_{\pi(p)} f_{\pi(p)}(p)=\left\langle\overline{k(p, \cdot)}, A_{\pi(p)} f_{\pi(p)}\right\rangle_{\pi(p)},
$$


where $\left\langle f_{1}, f_{2}\right\rangle_{\omega}=\int_{\mathcal{X}^{\omega}} \overline{f_{1}(q)} f_{2}(q) d \alpha^{\omega}(q)$. This shows that $K A$ has a kernel $k_{K A}$ given by

$$
\overline{k_{K A}(p, q)}=\left(A_{\pi(p)}^{*} \overline{k(p, \cdot)}\right)(q),
$$

which finishes the proof.

For a Carleman operator $K$ the expressions $\tau\left(K K^{*}\right)$ and $\tau\left(K^{*} K\right)$ can directly be calculated.

Proposition 4.5. Let $K \in \mathcal{K}$ be given. Then we have

$$
\tau\left(K^{*} K\right)=\int_{\Omega} \int_{\mathcal{X}^{\omega}} \int_{\mathcal{X}^{\omega}} u(q)|k(p, q)|^{2} d \alpha^{\omega}(p) d \alpha^{\omega}(q) d \mu(\omega)=\tau\left(K K^{*}\right),
$$

for any $u \in \mathcal{F}^{+}(\mathcal{X})$ satisfying $\nu * u \equiv 1$.

Proof. We use the well known formula $\operatorname{tr}\left(A^{*} A\right)=\int_{S \times S}|a(x, y)|^{2} d \lambda(x) d \lambda(y)$ valid for any bounded operator $A$ with kernel $a$. Note that this formula holds for both $a \in L^{2}(S \times S, \lambda \otimes \lambda)$ and $a \notin L^{2}(S \times S, \lambda \otimes \lambda)$. In the latter case both sides of the formula are just infinity. Now, we can calculate as follows:

$$
\begin{aligned}
\tau\left(K^{*} K\right) & =\int_{\Omega} \operatorname{tr}\left(M_{u_{\omega}}^{1 / 2} K_{\omega}^{*} K_{\omega} M_{u_{\omega}}^{1 / 2}\right) d \mu(\omega) \\
& =\int_{\Omega} \int_{\mathcal{X}^{\omega}} \int_{\mathcal{X}^{\omega}} u(q)|k(p, q)|^{2} d \alpha^{\omega}(p) d \alpha^{\omega}(q) d \mu(\omega) .
\end{aligned}
$$

This proves the first equation. To show the second equation, we insert $\nu * u \equiv 1$ in the first equation, finding

$\tau\left(K^{*} K\right)=\int_{\Omega} \int_{\mathcal{X}^{\omega}} \int_{\mathcal{X}^{\omega}}\left(\int_{\mathcal{G}^{\omega}} u\left(g^{-1} p\right) d \nu^{\omega}(g)\right) u(q)|k(p, q)|^{2} d \alpha^{\omega}(q) d \alpha^{\omega}(p) d \mu(\omega)$.

By Fubini and the fact that $K$ is invariant, we infer

$$
\cdots=\int_{\mathcal{G}} \int_{\mathcal{X}^{r(g)}} \int_{\mathcal{X}^{r(g)}} u\left(g^{-1} p\right) u(q)\left|k\left(g^{-1} p, g^{-1} q\right)\right|^{2} d \alpha^{r(g)}(p) d \alpha^{r(g)}(q) d(\mu \circ \nu)(g) \text {. }
$$

Invoking invariance of $\mu$, i.e. $(\mu \circ \nu)^{\sim}=\mu \circ \nu$, we finally obtain

$$
\begin{aligned}
\cdots & =\int_{\mathcal{G}} \int_{\mathcal{X}^{s(g)}} \int_{\mathcal{X}^{s(g)}} u(g p) u(q)|k(g p, g q)|^{2} d \alpha^{s(g)}(p) d \alpha^{s(g)}(q) d(\mu \circ \nu)(g) \\
& =\int_{\mathcal{G}} \int_{\mathcal{X}^{r(g)}} \int_{\mathcal{X}^{r(g)}} u\left(p^{\prime}\right) u\left(g^{-1} q^{\prime}\right)\left|k\left(p^{\prime}, q^{\prime}\right)\right|^{2} d \alpha^{r(g)}\left(p^{\prime}\right) d \alpha^{r(g)}\left(q^{\prime}\right) d(\mu \circ \nu)(g) .
\end{aligned}
$$

Now, the desired equality follows by reversing the steps from the preceding calculation. This finishes the proof.

The proposition shows that $\tau$ has the trace property $\tau\left(A A^{*}\right)=\tau\left(A^{*} A\right)$ for all Carleman operators $A$. To show that $\tau$ is actually a trace, we need two more steps. In the first step, we show that $\tau$ must be a trace if it satisfies this trace property for sufficiently many operators. In the second step, we show that the set of Carleman operators is large. 
Lemma 4.6. Let $\mathcal{I}$ be a right ideal in a von Neumann algebra $\mathcal{N}$ acting on a separable Hilbert space $\mathcal{H}$ with $\overline{\mathcal{I}^{*} \mathcal{I}(\mathcal{H})}=\mathcal{H}$. Let $\tau$ be a normal weight on $\mathcal{N}$ satisfying $\tau\left(A^{*} A\right)=\tau\left(A A^{*}\right)$ for all $A \in \mathcal{I}$. Then $\tau$ is a trace.

Proof. It suffices to show $\tau\left(U A U^{*}\right)=\tau(A)$ for arbitrary $A \in \mathcal{N}_{+}$and unitary $U \in \mathcal{N}$. Let $C(\mathcal{I})$ be the norm closure of $\mathcal{I}^{*} \mathcal{I}$. Obviously, $\mathcal{I}^{*} \mathcal{I}$ is a dense ideal in $C(\mathcal{I})$. By [Dix77, Prop. 1.7.2], there exists then an increasing approximate identity $\left(I_{\lambda}\right)$ for $C(\mathcal{I})$ in $\mathcal{I}$. As $\mathcal{H}$ is separable, we can choose $\left(I_{\lambda}\right)$ to be a sequence $\left(I_{n}\right)$. By $\overline{\mathcal{I}^{*} \mathcal{I}(\mathcal{H})}=\mathcal{H}$, we infer that $I_{n}$ converges monotonously to the identity $\operatorname{Id}$ on $\mathcal{H}$. By polarization, every $I_{n} \in \mathcal{I}^{*} \mathcal{I}$ can be written as

$$
I_{n}=\sum D_{i, n}^{*} D_{i, n}-\sum C_{j, n}^{*} C_{j, n}
$$

for suitable, finitely many $D_{i, n}, C_{j, n} \in \mathcal{I}$. Now, let an arbitrary $D \in \mathcal{I}$ be given. Using that $\mathcal{I}$ is a right ideal, we can calculate

$$
\tau\left(U A^{\frac{1}{2}} D^{*} D A^{\frac{1}{2}} U^{*}\right)=\tau\left(D A^{\frac{1}{2}} U^{*} U A^{\frac{1}{2}} D^{*}\right)=\tau\left(D A^{\frac{1}{2}} A^{\frac{1}{2}} D^{*}\right)=\tau\left(A^{\frac{1}{2}} D^{*} D A^{\frac{1}{2}}\right) .
$$

Combining (18),(17) and the fact that $\tau$ is normal, one can conclude the proof of the Lemma as follows: $\tau\left(U A U^{*}\right)=\lim _{n \rightarrow \infty} \tau\left(U A^{\frac{1}{2}} I_{n} A^{\frac{1}{2}} U^{*}\right)=$ $\lim _{n \rightarrow \infty} \tau\left(A^{\frac{1}{2}} I_{n} A^{\frac{1}{2}}\right)=\tau(A)$.

Proposition 4.7. Consider (RSM) and define, for $t \geq 0$, the operator $S(t): L^{2}(\Omega \times X) \rightarrow L^{2}(\Omega \times X)$, by $S(t) f_{\omega}(x):=\left(e^{-t \Delta_{\omega}} f_{\omega}\right)(x)$. Then, $S(t)$ is a selfadjoint bounded random operator for each $t \geq 0$. For $t>0$, the operator $S(t)$ belongs to $\mathcal{K}$. The family $t \mapsto S(t)$ is a strongly continuous semigroup.

Proof. Property (2) gives immediately the necessary measurability of $S(t)$. Moreover, $e^{-t \Delta_{\omega}}$ is a selfadjoint contraction for every $\omega \in \Omega$. Therefore, Fubini easily shows $\|S(t) f\| \leq\|f\|$ for every $f \in L^{2}(\Omega \times X)$. Thus, $S(t)$ is a decomposable operator. As its fibres are selfadjoint, it is selfadjoint as well (cf. [RS78, Thm. XIII.85] and [Dix77, Appendix A.78]). The transformation formula $\Delta_{\omega}=U_{(\omega, \gamma)} \Delta_{\gamma^{-1} \omega} U_{(\omega, \gamma)}^{*}$ shows that $S(t)$ satisfies the equivariance property. Thus, $S(t)$ is indeed a random operator. Using that $t \mapsto e^{-t \Delta_{\omega}}$ is a strongly continuous semigroup of operators with norm not exceeding 1 for every $\omega \in \Omega$, we can directly calculate that $S(t)$ is a strongly continuous semigroup, as well.

For $t>0$, every $S(t)$ has a kernel $k_{\omega}^{t}$ (see, e.g., Cha84 or [SchY94]). Using selfadjointness and the semigroup property, we can calculate

$$
\int_{X}\left|k_{\omega}^{t}(x, y)\right|^{2} d \lambda^{\omega}(y)=\int_{X} k_{\omega}^{t}(x, y) k_{\omega}^{t}(y, x) d \lambda^{\omega}(y)=k_{\omega}^{2 t}(x, x)<\infty .
$$

This shows that $S(t)$ belongs to $\mathcal{K}$.

Now, the following theorem is not hard to prove.

Theorem 4.8. In the example (RSM), the map $\tau$ is a normal trace. 
Proof. By Proposition 4.5 and Lemma 4.6, it suffices to show that $\mathcal{K}^{*} \mathcal{K} L^{2}(X \times$ $\Omega)$ is dense in $L^{2}(\Omega \times X)$. But, this follows from the foregoing proposition, which shows that $S(t) f$ converges to $f$ for every $f \in L^{2}(\Omega \times X)$, where $S(t) \in \mathcal{K}^{*} \mathcal{K}$ by the semigroup property.

\section{Fundamental RESUlts FOR RANDOM OPERATORS}

In this section we present a comprehensive treatment of the basic features $(\mathrm{P} 1)-(\mathrm{P} 4)$ of random operators mentioned in the introduction. This unifies and extends the corresponding known results about random Schrödinger operators in Euclidean space and random operators induced by tilings. As in the previous section, we always assume that $\left(\mathcal{G}, \nu, \mu, \mathcal{X}, \alpha, u_{0}\right)$ is an admissible setting.

A function $f$ on $\Omega$ is called invariant if $f \circ r=f \circ s$. The groupoid $\mathcal{G}$ is said to be ergodic (with respect to $\mu$ ) if every invariant measurable function $f$ is $\mu$-almost everywhere constant. This translates, in the particular case (RSM), to an ergodic action of $\Gamma$ on $\Omega$. We will be mostly concerned with decomposable selfadjoint operators on $L^{2}(\mathcal{X}, \mu \circ \alpha)$.

For a selfadjoint operator $H$, we denote by $\sigma_{d i s c}(H), \sigma_{e s s}(H), \sigma_{a c}(H), \sigma_{s c}(H)$ and $\sigma_{p p}(H)$ the discrete, essential, absolutely continuous, singular continuous, and pure point part of its spectrum respectively.

Theorem 5.1. Let $\mathcal{G}$ be an ergodic groupoid. Let $H=\int_{\Omega}^{\oplus} H_{\omega} d \mu(\omega)$ be a selfadjoint operator affiliated to $\mathcal{N}(\mathcal{G}, \mathcal{X})$. There exist $\Omega^{\prime} \subset \Omega$ of full measure and $\Sigma, \Sigma \bullet \subset \mathbb{R}, \bullet=$ disc, ess, ac, sc,pp, such that

$$
\sigma\left(H_{\omega}\right)=\Sigma, \quad \sigma_{\bullet}\left(H_{\omega}\right)=\Sigma \bullet \quad \text { for all } \omega \in \Omega^{\prime}
$$

for $\bullet=$ disc, ess, ac, sc, pp. Moreover, $\sigma(H)=\Sigma$.

Note that $\sigma_{p p}$ denotes the closure of the set of eigenvalues.

Proof. The proof is essentially a variant of well known arguments (cf. e.g. KS79, KM82b, Car86, CFSK87, CL90). However, as our stetting is different and, technically speaking, involves direct integrals with non constant fibres, we sketch a proof.

Let $\mathcal{J}$ be the family of finite unions of open intervals in $\mathbb{R}$, all of whose endpoints are rational. Let $\mathcal{J}^{k}$ consist of those elements of $\mathcal{J}$ which are unions of exactly $k$ intervals. Denote the spectral family of a selfadjoint operator $H$ by $E_{H}$. It is not hard to see that $\omega \mapsto \operatorname{tr} E_{H_{\omega}}(B)$ is an invariant measurable function for every $B \in \mathcal{J}$ (and, in fact, for every Borel measurable $B \subset \mathbb{R}$ ). Thus, by ergodicity, this map is almost surely constant. Denote this almost sure value by $f_{B}$. As $\mathcal{J}$ is countable, we find $\Omega^{\prime} \subset \Omega$ of full measure, such that for every $\omega \in \Omega^{\prime}$, we have $\operatorname{tr} E_{H_{\omega}}(B)=f_{B}$ for every $B \in \mathcal{J}$. By

$$
\sigma(H)=\left\{\lambda \in \mathbb{R}: E_{H}(B) \neq 0, \text { for all } B \in \mathcal{J} \text { with } \lambda \in B\right\},
$$

and as $\operatorname{tr}$ is faithful, we infer constancy of $\sigma\left(H_{\omega}\right)$ on $\Omega^{\prime}$. By a completely analogous argument, using $\sigma_{\text {ess }}(H)=\left\{\lambda \in \mathbb{R}: \operatorname{tr} E_{H}(B)=\right.$ 
$\infty$ for all $B \in \mathcal{I}$ with $\lambda \in B$ \}, we infer almost sure constancy of $\sigma_{e s s}\left(H_{\omega}\right)$ and thus also of $\sigma_{\text {disc }}\left(H_{\omega}\right)$.

To show constancy of the remaining spectral parts, it suffices to show measurability of

$$
\omega \mapsto\left\langle g_{\omega}, E_{H_{\omega}}^{p p}(B) g_{\omega}\right\rangle_{\omega}, \text { and } \omega \mapsto\left\langle g_{\omega}, E_{H_{\omega}}^{\text {sing }}(B) g_{\omega}\right\rangle_{\omega}
$$

for every $g \in L^{2}(X, \mu \circ \alpha)$ and every $B \in \mathcal{J}$. Here, of course, $E_{H}^{p p}$ and $E_{H}^{\text {sing }}$ denote the restrictions of the spectral family to the pure point and singular part of the underlying Hilbert space, respectively. To show these measurabilities, recall that for an arbitrary measure $\mu$ on $\mathbb{R}$ with pure point part $\mu^{p p}$ and singular part $\mu^{\text {sing }}$ we have

$\mu^{\text {sing }}(B)=\lim _{n \rightarrow \infty} \sup _{J \in \mathcal{J},|J| \leq n^{-1}} \mu(B \cap J), \mu^{p p}(B)=\lim _{k \rightarrow \infty} \lim _{n \rightarrow \infty} \sup _{J \in \mathcal{J}^{k},|J| \leq n^{-1}} \mu(B \cap J)$.

Here, the first equation was proven by Carmona (see [Car86, CL90, CFSK87]), and the second follows by a similar argument. As this latter reasoning does not seem to be in the literature, we include a discussion in Appendix B. Given these equalities, (20) is an immediate consequence of measurability of $\omega \mapsto$ $\left\langle g_{\omega}, E_{H_{\omega}}(B) g_{\omega}\right\rangle_{\omega}$, (which holds by assumption on $H$ ). (Note that instead of considering $\mu^{p p}$ as above, one could have considered the continuous part $\mu^{c}$ of $\mu$ by a method given in CL90.)

It remains to show the last statement. Obviously, $E_{H}(B)=0$ if and only if $E_{H_{\omega}}(B)=0$ for almost every $\omega \in \Omega$. Using this, (19), and almost sure constancy of $\operatorname{tr} E_{H_{\omega}}(B)$, infer that $\lambda \in \sigma(H)$ if and only if $E_{H_{\omega}}(B) \neq 0$ for every $B \in \mathcal{J}$ with $\lambda \in B$ and almost every $\omega \in \Omega$. This proves the last statement. Alternatively, one could follow the proof of [RS78, Thm.XIII.85] which is valid in the case of non-constant fibres, too.

Recall that a measure $\phi$ on $\mathbb{R}$ is a spectral measure for a selfadjoint operator $H$ with spectral family $E_{H}$ if, for Borel measurable $B \subset \mathbb{R}, \phi(B)=0 \Leftrightarrow E_{H}(B)=$ 0 .

Proposition 5.2. For a Borel measurable $B$ in $\mathbb{R}$ and a selfadjoint $H$ affiliated to $\mathcal{N}(\mathcal{G}, \mathcal{X})$, let $\rho_{H}(B)$ be defined by $\rho_{H}(B):=\tau\left(E_{H}(B)\right)$. Then $\rho_{H}$ is a spectral measure for $H$. Moreover, for a bounded measurable function $F: \mathbb{R} \longrightarrow[0, \infty)$, the equality $\tau(F(H))=\rho_{H}(F):=\int F(x) d \rho_{H}(x)$ holds.

Proof. As $\tau$ is a normal weight, $\rho_{H}$ is a measure. As $\tau$ is faithful, $\rho_{H}$ is a spectral measure. The last statement is then immediate for linear combinations of characteristic functions with non negative coefficients. For arbitrary functions it then follows after taking suitable monotone limits and using normality of $\tau$.

Definition 5.3. The measure $\rho_{H}$ is called (abstract) density of states.

Corollary 5.4. The topological support $\operatorname{supp}\left(\rho_{H}\right)=\left\{\lambda \in \mathbb{R} \mid \rho_{H}(] \lambda-\epsilon, \lambda+\epsilon[)>\right.$ 0 for all $\epsilon>0\}$ coincides with the spectrum $\sigma(H)$ of $H$ for every selfadjoint $H$ 
affiliated to $\mathcal{N}(\mathcal{G}, \mathcal{X})$. If $\mathcal{G}$ is, furthermore, ergodic this gives $\operatorname{supp}\left(\rho_{H}\right)=\sigma\left(H_{\omega}\right)$ for almost every $\omega \in \Omega$.

Proof. The first statement is immediate as $\rho_{H}$ is a spectral measure. The second follows from Theorem 5.1 .

Remark 5.5. In general, $\rho_{H}$ is not the spectral measure of the fibre $H_{\omega}$. Actually, there are several examples where the set

$$
\Omega^{\prime}:=\left\{\omega \in \Omega \mid \rho_{H} \text { is a spectral measure for the operator } H_{\omega}\right\}
$$

has measure zero. We shortly discuss two classes of them (see AS83] as well for related material).

Firstly we consider the case where $\left(H_{\omega}\right)_{\omega}$ exhibits localization in an energy interval $I$. This means that for a set $\Omega_{l o c} \subset \Omega$ of full measure

$$
\sigma\left(H_{\omega}\right) \cap I \neq \emptyset \text { and } \sigma_{c}\left(H_{\omega}\right) \cap I=\emptyset
$$

for all $\omega \in \Omega_{l o c}$. Examples of such ergodic, random operators on $L^{2}\left(\mathbb{R}^{d}\right)$ and $\ell^{2}\left(\mathbb{Z}^{d}\right)$ can be found e.g. in the textbooks [CL90, PF92, Sto01]. They particularly include random Schrödinger operators in one- and higher dimensional configuration space. If $H_{\omega}$ is an ergodic family of Schrödinger operators on $\ell^{2}\left(\mathbb{Z}^{d}\right)$ or $L^{2}(\mathbb{R})$ one knows moreover for all energy values $E \in \mathbb{R}$

$$
\mathbb{P}\{\Omega(E)\}=0, \text { where } \Omega(E):=\left\{\omega \in \Omega \mid E \text { is an eigenvalue of } H_{\omega}\right\} .
$$

Assume that $\rho_{H}$ is a spectral measure of $H_{\omega}$ for some $\omega \in \Omega_{l o c}$. Then there exists an eigenvalue $E \in I$ of $H_{\omega}$ and consequently $\rho_{H}(\{E\})>0$. Thus $\rho_{H}$ can only be a spectral measure for $H_{\omega^{\prime}}$ if $\omega^{\prime} \in \Omega(E)$, but this set has measure zero.

Similarly, there are one-dimensional discrete random Schrödinger operators which have purely singular continuous spectrum almost surely. An example is the almost Mathieu operator, cf. AS83, for a certain range of parameters. Moreover, in GJLS99] it is proven that the singular continuous components of the spectral measures of these models are almost surely pairwise orthogonal. Thus again, $\rho_{H}$ can be a spectral measure only for a set of $\omega$ of measure zero.

Lemma 5.6. Let $\mathcal{G}$ be ergodic with respect to $\mu$ with $\mu(\Omega)<\infty$ such that the following exhaustion property holds:

$$
\begin{aligned}
& \text { There exists a sequence }\left(f_{n}\right) \text { in } \mathcal{F}^{+}(\mathcal{G}) \text { with } \mathcal{G}=\bigcup_{n}\left\{g: f_{n}(g)>0\right\}, \\
& \left\|f_{n}\right\|_{\infty} \rightarrow 0 \text { as } n \rightarrow \infty \text { and } \nu\left(\widetilde{f_{n}}\right) \equiv 1 \text { for all } n \in \mathbb{N} \text {. }
\end{aligned}
$$

Then, for every transverse function $\phi$, either $\phi^{\omega}(1) \equiv 0$ almost surely or $\phi^{\omega}(1) \equiv \infty$ almost surely.

Proof. By ergodicity, $\phi^{\omega}(1)$ is constant almost surely. Assume $\phi^{\omega}(1)=c<\infty$ for almost every $\omega$. Lemma 2.9 (a) implies that $\int_{\Omega} \phi^{\omega}(f) d \mu(\omega)$ is independent of $f \in \mathcal{F}^{+}$with $\nu(\widetilde{f}) \equiv 1$. For such $f$, we infer

$$
\text { Constant }=\int_{\Omega} \phi^{\omega}(f) d \mu \leq\|f\|_{\infty} c \mu(\Omega) .
$$


As this is in particular valid for every $f_{n}, n \in \mathbb{N}$, we infer Constant $=0$. This shows $\phi^{\omega}\left(f_{n}\right)=0$ for almost every $\omega$ and every $n \in \mathbb{N}$. By $\mathcal{G}=\bigcup_{n}\left\{g: f_{n}(g)>\right.$ $0\}$, this gives $\phi^{\omega}(1)=0$ for almost every $\omega \in \Omega$.

Remark 5.7. Let $(\mathcal{G}, \nu, \mu)$ be a measurable groupoid, $(\mathcal{X}, \alpha)$ be an associated random variable and $u \in \mathcal{F}^{+}(\mathcal{X})$ such that property (6) holds and $\nu * u \equiv 1$. Let $f_{n}$ be a sequence satisfying condition (21). Then $\left(\mathcal{G}, \nu, \mu, \mathcal{X}, \alpha, u_{0}\right)$ with $u_{0}=q_{u}\left(\sum_{n=1}^{\infty} \frac{1}{2^{n}} f_{n}\right)$ is an admissible setting.

Remark 5.8. The exhaustion property (21) can easily be seen to hold in the case (RSM), if $\Gamma$ is infinite. Namely, we can choose

$$
f_{n}(g)=f_{n}(\omega, \gamma)=\frac{1}{\left|I_{n}\right|} \chi_{I_{n}}(\gamma)
$$

Here, $I_{n}$ is an exhaustion of the group $\Gamma$.

The lemma has an interesting spectral consequence.

Corollary 5.9. Let the assumptions of Lemma 5.6 be satisfied and the selfadjoint $H=\int_{\Omega}^{\oplus} H_{\omega} d \mu(\omega)$ be affiliated to $\mathcal{N}(\mathcal{G}, \mathcal{X})$. Then $H_{\omega}$ has almost surely no discrete spectrum.

Proof. We already know that the discrete spectrum is constant almost surely. Let $B$ be an arbitrary Borel measurable subset of $\mathbb{R}$. Then, the map $\omega \mapsto \operatorname{tr}\left(E_{H_{\omega}}(B) M_{q_{\omega}(\cdot)}\right)$ is a transverse function, by Lemma 4.1 (a). Thus, $\operatorname{tr}\left(E_{H_{\omega}}(B) M_{q_{\omega}(1)}\right)=\operatorname{tr}\left(E_{H_{\omega}}(B)\right)$ equals almost surely zero or infinity. As $B$ is arbitrary, this easily yields the statement.

Remark 5.10. Corollary 5.9 is well known for certain classes of random operators. However, our proof of the key ingredient, Lemma 5.6, is new. It seems to be more general and more conceptual. Moreover, as discussed in the proof of Theorem 5.11 below, Lemma 5.6 essentially implies that $\mathcal{N}(\mathcal{G}, \mathcal{X})$ is not type $I$. Thus, the above considerations establish a connection between the absence of discrete spectrum and the type of the von Neumann algebra $\mathcal{N}(\mathcal{G}, \mathcal{X})$.

Let us finish this section by discussing factorial and type properties of $\mathcal{N}(\mathcal{G}, \mathcal{X})$. By [Con79, Cor. V.8] (cf. Cor. V.7 of [Con79], as well), the von Neumann algebra $\mathcal{N}$ is a factor (i.e. satisfies $\mathcal{N} \cap \mathcal{N}^{\prime}=\mathbb{C}$ Id) if $\mathcal{G}$ is ergodic with respect to $\mu$ and the freeness condition (14) holds.

There are three different types of factors. These types can be introduced in various ways. We will focus on an approach centered around traces (cf. Con94] for further discussion and references).

A factor is said to be of type $I I I$, if it does not admit a semifinite normal trace. If a factor admits such a trace, then this trace must be unique (up to a multiplicative constant) and there are two cases. Namely, either, this trace assumes only a discrete set of values on the projections, or the range of the trace on the projections is an interval of the form $[0, a]$ with $0<a \leq \infty$. In the 
first case, the factor is said to be of type $I$. It must then be isomorphic to the von Neumann algebra of bounded operators on a Hilbert space. In the second case, the factor is said to be of type $I I$.

Theorem 5.11. Let the assumptions of Lemma 5.6 and condition (14) be satisfied, then $\mathcal{N}(\mathcal{G}, \mathcal{X})$ is a factor of type $I I$.

Proof. By Lemma 5.6, there does not exist a bounded transversal function $\phi$ whose support $\left\{\omega: \phi^{\omega}(1) \neq 0\right\}$ has positive $\mu$ measure. By [Con79, Cor. V.9], we infer that $\mathcal{N}(\mathcal{G}, \mathcal{X})$ is not type $I$. On the other hand, as $\tau$ is a semifinite normal trace on $\mathcal{N}$ by Theorem 4.2 , it is not type $I I I$.

Remark 5.12. (a) In the case (RSM), under the countability and freeness assumptions (6) and (14), we know that $\mathcal{N}(\Omega \times \Gamma, \Omega \times X)$ is actually a factor of type $I I_{\infty}$ : Since the identity on an infinite dimensional Hilbert space has trace equal to infinity, we conclude

$$
\operatorname{tr}_{L^{2}(X)}\left(M_{\chi_{\mathcal{D}}}\right)=\operatorname{tr}_{L^{2}(\mathcal{D})}(\mathrm{Id})=\infty
$$

where $\mathcal{D}$ is a fundamental domain as in Remark 2.8, Now, choosing $u_{\omega}(x) \equiv$ $\chi_{\mathcal{D}}(x)$, we have $\nu * u \equiv 1$ and, consequently,

$$
\tau(\mathrm{Id})=\mathbb{E}\left\{\operatorname{tr}\left(M_{\chi_{\mathcal{D}}}\right)\right\}=\infty .
$$

(b) In the case of tiling groupoids and percolation models one finds factors of type $I I$ with a finite value of the canonical trace on the identity. This finite value is determined by geometric features of the underlying tiling, respectively the percolation process, cf. Sections 7 and 8 .

\section{The Pastur-ŠSubin-trace formula for (RSM)}

The aim of this section is to give an explicit exhaustion construction for the abstract density of states (cf. Section 5) of the model (RSM). The construction shows in particular that the IDS, the distribution function of the density of states, is self-averaging. This means that it can be expressed by a macroscopic limit which is $\omega$-independent, although one did note take the expectation over the randomness.

We recall in this section the relevant definitions and results of [LPV04] concerning the example (RSM) and outline the main steps of the proofs.

In the following we assume that the group $\Gamma$ is amenable to be able to apply an appropriate ergodic theorem. The exhaustion procedure yields a limiting distribution function which coincides, at all continuity points, with the distribution function of the abstract density of states. For the calculation of this distribution function the Laplace transformation has proved useful Pas71, Šub82. We refer to the second reference for a detailed description of the general strategy.

In Section 3 of [LPV04] the operators $H_{\omega}$ of (RSM) are defined via quadratic forms, the measurability of the latter is established, and the validity of the measurability condition (2) is deduced under the following additional hypotheses

(22) The map $\Omega \times T X \rightarrow \mathbb{R},(\omega, v) \mapsto g_{\omega}(v, v)$ is jointly measurable. 
(23) There is a $\left.C_{g} \in\right] 0, \infty[$ such that

$$
C_{g}^{-1} g_{0}(v, v) \leq g_{\omega}(v, v) \leq C_{g} g_{0}(v, v) \text { for all } v \in T X .
$$

(24) There is a $\left.C_{\rho} \in\right] 0, \infty[$ such that

$$
\left|\nabla_{0} \rho_{\omega}(x)\right|_{0} \leq C_{\rho} \text { for all } x \in X,
$$

where $\nabla_{0}$ denotes the gradient with respect to $g_{0}, \rho_{\omega}$ is the unique smooth density of $\lambda^{0}$ with respect to $\lambda^{\omega}$, and $|v|_{0}^{2}=g_{0}(v, v)$.

(25) There is a uniform lower bound $K \in \mathbb{R}$ for the Ricci curvatures of all Riemannian manifolds $\left(X, g_{\omega}\right)$. Explicitly, $\operatorname{Ric}\left(g_{\omega}\right) \geq K g_{\omega}$ for all $\omega \in \Omega$ and on the whole of $X$.

(26) Let $V: \Omega \times X \rightarrow \mathbb{R}$ be a jointly measurable mapping such that for all $\omega \in \Omega$ the potential $V_{\omega}:=V(\omega, \cdot) \geq 0$ is in $L^{1}(A)$ for any compact $A \subset X$.

A key technique is an ergodic theorem by Lindenstrauss [Lin01, valid for amenable groups $\Gamma$ acting ergodically by measure preserving transformations on $\Omega$. This theorem relies on suitable sequences $\left(I_{n}\right)_{n}, I_{n} \subset \Gamma$, so called tempered Følner sequences introduced by Shulman Shu88. For an appropriate fundamental domain $\mathcal{D}$ (cf. $\S 3$ in AS93), the sequence $\left(I_{n}\right)_{n}$ induces a sequence $\left(A_{n}\right)_{n}, A_{n} \subset X$ by $A_{n}=\operatorname{int}\left(\overline{\bigcup_{\gamma \in I_{n}} \gamma \mathcal{D}}\right)$. The ergodic theorem in Lin01 together with the equivariance property (1) imply that the following limits hold pointwise almost surely and in $L^{1}(\Omega, \mathbb{P})$ sense

$$
\begin{array}{r}
\lim _{n \rightarrow \infty} \frac{1}{\left|I_{n}\right|} \operatorname{tr}\left(\chi_{A_{n}} e^{-t H_{\omega}}\right)=\mathbb{E}\left\{\operatorname{tr}\left(\chi_{\mathcal{D}} e^{-t H_{\bullet}}\right)\right\} \\
\lim _{n \rightarrow \infty} \frac{1}{\left|I_{n}\right|} \lambda^{\omega}\left(A_{n}\right)=\mathbb{E}\left(\lambda^{\bullet}(\mathcal{D})\right) .
\end{array}
$$

Moreover the $L^{\infty}(\Omega, \mathbb{P})$ norms of the sequences

$$
\left(\frac{\operatorname{tr}\left(\chi_{A_{n}} e^{-t H_{\omega}}\right)}{\left|I_{n}\right|}\right)_{n}, \quad\left(\frac{\lambda^{\omega}\left(A_{n}\right)}{\left|I_{n}\right|}\right)_{n}, \quad\left(\frac{\left|I_{n}\right|}{\lambda^{\omega}\left(A_{n}\right)}\right)_{n}
$$

are uniformly bounded in the variable $n \in \mathbb{N}$.

Another important ingredient in the proof of (30) below is the following heat kernel lemma (see [LPV04, Lemma 7.2]): Let $\left(A_{n}\right)_{n}$ be as above. Then we have

$$
\lim _{n \rightarrow \infty} \sup _{\omega \in \Omega} \frac{1}{\lambda^{\omega}\left(A_{n}\right)}\left|\operatorname{tr}\left(\chi_{A_{n}} e^{-t H_{\omega}}\right)-\operatorname{tr}\left(e^{-t H_{\omega}^{n}}\right)\right|=0 .
$$

The sets $A_{n}$ together with the random family $\left(H_{\omega}\right)$ of Schrödinger operators on $X$ are used to introduce the normalized eigenvalue counting functions

$$
N_{\omega}^{n}(\lambda)=\frac{\left|\left\{i \mid \lambda_{i}\left(H_{\omega}^{n}\right)<\lambda\right\}\right|}{\lambda^{\omega}\left(A_{n}\right)}
$$

where $H_{\omega}^{n}$ denotes the restriction of $H_{\omega}=\Delta+V_{\omega}$ to the domain $A_{n}$ with Dirichlet boundary conditions and $\lambda_{i}\left(H_{\omega}^{n}\right)$ denotes the $i$-th eigenvalue of $H_{\omega}^{n}$ counted with multiplicities. The cardinality of a set is denoted by $|\cdot|$. Note that 
$\operatorname{tr}\left(e^{-t H_{\omega}^{n}}\right)=\int e^{-t \lambda} d N_{\omega}^{n}(\lambda)$. Using (27), (28) and (29) it is shown in LPV04] that there exists a distribution function $N_{H}: \mathbb{R} \rightarrow\left[0, \infty\left[\right.\right.$, i.e., $N_{H}$ is left continuous and monotone increasing, such that the following $\mathbb{P}$-almost sure pointwise and $L^{1}$-convergence of Laplace-transforms holds true: for all $t>0$

$$
\lim _{n \rightarrow \infty} \tilde{N}_{\omega}^{n}(t)=\lim _{n \rightarrow \infty} \int_{-\infty}^{\infty} e^{-t \lambda} d N_{\omega}^{n}(\lambda)=\int_{-\infty}^{\infty} e^{-t \lambda} d N_{H}(\lambda)=\tilde{N}_{H}(t),
$$

and that $\tilde{N}_{H}$ can be identified with the explicit expression

$$
\tilde{N}_{H}(t)=\frac{\mathbb{E}\left(\operatorname{tr}\left(\chi_{\mathcal{D}} e^{-t H \bullet}\right)\right)}{\mathbb{E}\left(\lambda^{\bullet}(\mathcal{D})\right)}=\frac{\tau\left(e^{-t H}\right)}{\mathbb{E}\left(\lambda^{\bullet}(\mathcal{D})\right)}
$$

This implies by the Pastur-Šubin-Lemma [Pas71, ك̌ub79] the following convergence (for $\mathbb{P}$-almost all $\omega \in \Omega$ )

$$
\lim _{n \rightarrow \infty} N_{\omega}^{n}(\lambda)=N_{H}(\lambda)
$$

at all continuity points of $N_{H}$. Note that $N_{H}$ does not depend on $\omega$. Moreover, $N_{H}$ does not depend on the sequence $\left(A_{n}\right)_{n}$ as long as $\left(A_{n}\right)_{n}$ is chosen in the above way. The function $N_{H}$ is called the integrated density of states.

Now, our trace formula reads as follows.

Theorem 6.1. Let the measure $\rho_{H}$ be the abstract density of states introduced in Section 5. Then we have

$$
N_{H}(\lambda)=\frac{\rho_{H}(]-\infty, \lambda[)}{\mathbb{E}(\lambda \bullet(\mathcal{D}))}
$$

at all continuity points $\lambda$ of $N_{H}$.

Remark 6.2. The theorem implies in particular

$$
N_{H}(\lambda)=\frac{1}{\mathbb{E}\left(\lambda^{\bullet}(\mathcal{D})\right)} \mathbb{E}\left\{\operatorname{tr}\left(\chi_{\mathcal{D}} E_{H_{\omega}}(]-\infty, \lambda[)\right)\right\},
$$

where $\mathcal{D}$ is a fundamental domain of $\Gamma$ as in Remark 2.8. This alternative localized formula for the IDS is well-known in the Euclidean case. Note that it doesn't rely on a choice of boundary condition.

Proof. By the uniqueness lemma for the Laplace transform (see Lemma C.1 in the Appendix) it suffices to show that for all $t>0$

$$
\mathbb{E}\left(\lambda^{\bullet}(\mathcal{D})\right) \int e^{-t \lambda} d N_{H}(\lambda)=\int e^{-t \lambda} d \rho_{H}(\lambda) .
$$

To this end we observe that by (31) $\tau\left(e^{-t H}\right)=\mathbb{E}\left(\lambda^{\bullet}(\mathcal{D})\right) \int e^{-t \lambda} d N_{H}(\lambda)$ which leaves to prove that

$$
\tau\left(e^{-t H}\right)=\rho_{H}\left(e^{-t \lambda}\right) .
$$

To do so, we will use that the operator $H$ is bounded below, say $H \geq C$, with a suitable $C \in \mathbb{R}$. Define $F: \mathbb{R} \longrightarrow\left[0, \infty\left[\right.\right.$ by $F(\lambda)=e^{-t \lambda}$ if $\lambda \geq C$ and by $F(\lambda)=0$ otherwise. By spectral calculus, we infer $e^{-t H}=F(H)$ and, in particular, $\tau\left(e^{-t H}\right)=\tau(F(H))$. By Proposition [5.2, this implies $\rho_{H}(F)=$ 
$\tau\left(e^{-t H}\right)$. As, again by Proposition [5.2, $\rho_{H}$ is a spectral measure for $H$, its support is contained in $\left[C, \infty\left[\right.\right.$ and we easily find $\rho_{H}\left(e^{-t \lambda}\right)=\rho_{H}(F)$. Combining these equalities, we end up with the desired equality (34).

\section{QuASicRYSTAL MODElS}

In this section we shortly discuss how to use the above framework to study random operators associated to quasicrystals. Quasicrystals are usually modelled by tilings or Delone sets and these two approaches are essentially equivalent. Here, we work with Delone sets and follow LS01, LS04, LS03, to which we refer for further details. The investigation of quasicrystals via groupoids goes back to Kellendonk [Kel95, Kel97] and his study of K-theory and gap labelling in this context (see [BHZ00, LS01, LS04, LS03, for further discussion of quasicrystal groupoids and [BKL01, BOH01, BBG01, KP03] for recent work proving the so-called gap-labelling conjecture).

A subset $\omega$ of $\mathbb{R}^{d}$ is called Delone if there exist $0<r \leq R$ such that $r \leq\|x-y\|$ whenever $x, y \in \omega$ with $x \neq y$ and $\omega \cap\{y:\|y-x\| \leq R\} \neq \emptyset$ for all $x \in \mathbb{R}^{d}$. Here, $\|\cdot\|$ denotes the Euclidean norm on $\mathbb{R}^{d}$.

There is a natural action $T$ of $\mathbb{R}^{d}$ on the set of all Delone sets by translation (i.e. $T_{t} \omega=t+\omega$ ). Moreover, there is a topology (called the natural topology by some authors) such that $T$ is continuous. Then, $(\Omega, T)$ is called a Delone dynamical system if $\Omega$ is a compact $T$-invariant set of Delone sets.

In this case $\mathcal{G}(\Omega, T):=\Omega \times \mathbb{R}^{d}$ is clearly a groupoid with transversal function $\nu$ with $\nu^{\omega}=$ Lebesgue measure for all $\omega \in \Omega$. If $\mu$ is a $T$-invariant measure on $\Omega$, it is an invariant measure on $\mathcal{G}(\Omega, T)$ in the sense discussed above. By the compactness of $\Omega$ there exists at least one such non trivial $\mu$ by the KrylovBogolyubov theorem. In fact, in the prominent examples for quasicrystals, there is a unique such probability measure; these systems are called uniquely ergodic. This notation comes from the fact that this unique $T$-invarant measure is necessarily ergodic.

We now assume that $(\Omega, T)$ with invariant measure $\mu$ is given. Then, there is a natural space $\mathcal{X}$ given by

$$
\mathcal{X}=\{(\omega, x) \in \mathcal{G}(\Omega, T): x \in \omega\} \subset \mathcal{G}(\Omega, T) .
$$

Then, $\mathcal{X}$ inherits a topology from $\mathcal{G}(\Omega, T)$ and is in fact a closed subset. The space $\mathcal{X}$ is fibred over $\Omega$ with fibre map

$$
\pi: \mathcal{X} \longrightarrow \Omega,(\omega, x) \mapsto \omega .
$$

Thus, the fibre $\mathcal{X}^{\omega}$ can naturally be identified with $\omega$. In particular, every $g=(\omega, x) \in \mathcal{G}(\Omega, T)$ gives rise to a isomorphism $J(g): \mathcal{X}^{s(g)} \rightarrow \mathcal{X}^{r(g)}, J(g)(\omega-$ $x, p)=(\omega, p+x)$ and $J\left(g_{1} g_{2}\right)=J\left(g_{1}\right) J\left(g_{2}\right)$ and $J\left(g^{-1}\right)=J(g)^{-1}$. Each fibre $\omega$ carries the discrete measure $\alpha^{\omega}$ giving the weight one to each point of $\omega$. Then, $(\mathcal{X}, \alpha)$ is a random variable. 
Let furthermore $u \geq 0$ be a continuous function on $\mathbb{R}^{d}$ with $\int u(t) d t=1$. Then, $u$ gives rise to a function $u_{0}$ on $\mathcal{X}$ via

$$
u_{0}(\omega, x)=u(x)
$$

and

$$
\int_{\mathcal{G}(\Omega, T)^{\pi(p)}} u_{0}\left(\gamma^{-1} p\right) d \nu^{\pi(p)}(\gamma)=\int_{\mathbb{R}^{d}} u(t) d t=1 .
$$

Therefore, we are in an admissible setting.

The freeness condition that $\gamma^{-1} \omega \neq \omega$ whenever $\gamma \neq(\varepsilon, \omega)$ is known as aperiodicity.

The associated operators are given by families $A_{\omega}: \ell^{2}(\omega) \longrightarrow \ell^{2}(\omega), \omega \in \Omega$ satisfying a measurability and boundedness assumption as well as the equivariance condition

$$
A_{\omega}=U_{(\omega, t)} A_{\omega-t} U_{(\omega, t)}^{*}
$$

for $\omega \in \Omega$ and $t \in \mathbb{R}^{d}$, where $U_{(\omega, t)}: \ell^{2}(\omega-t) \longrightarrow \ell^{2}(\omega)$ is the unitary operator induced by translation.

There is a canonical trace on these operators given by

$$
\tau(A):=\int_{\Omega} \operatorname{tr}\left(M_{u_{0}} A_{\omega}\right) d \mu(\omega) .
$$

It is not hard to see that $\tau(\mathrm{Id})<\infty$ as there exists $r>0$ with $\|x-y\| \geq r$ whenever $x, y \in \omega$ for $\omega \in \Omega$ and $x \neq y$. If $\mu$ is ergodic, then $\tau$ (Id) is just the density of points of almost all $\omega \in \Omega$. In this case, we can conclude from the discussion in Section 5 that the discrete spectrum is absent. The necessary sequence $f_{n}$ is defined by

$$
f_{n}(\omega, x)=\frac{1}{\operatorname{vol}\left(B_{n}\right)} \chi_{B_{n}}(x),
$$

where $B_{n}$ is the ball in $\mathbb{R}^{d}$ around the origin with radius $n, \chi$ denotes the characteristic function and vol stands for Lebesgue measure.

In fact, assuming ergodicity of $\mu$ together with aperiodicity, i.e. freeness, we can even conclude from Section 5 that the von Neumann algebra of random operators is a factor of type $I I_{1}$.

Of course, in the ergodic case the results of Section 5 can be applied. They give almost sure constancy of the spectral components and the possibility to express the spectrum of a random operator $A$ with the help of the measure $\rho_{A}$ defined there by $\rho_{A}(\varphi)=\tau(\varphi(A))$ for continuous $\varphi$ on $\mathbb{R}$ with compact support.

Theorem 7.1. Let $\left(A_{\omega}\right)$ be a selfadjoint random operator in the setting discussed in this section and asume that $\mu$ is ergodic. Then there exists $\Omega^{\prime} \subset \Omega$ of full measure and subsets of the real numbers $\Sigma$ and $\Sigma_{\bullet}$, where - $\in\{$ disc, ess, ac, sc, $p p\}$, such that for all $\omega \in \Omega^{\prime}$

$$
\sigma\left(A_{\omega}\right)=\Sigma \quad \text { and } \quad \sigma_{\bullet}\left(A_{\omega}\right)=\Sigma \bullet
$$

for any $\bullet=$ disc, ess, ac, sc, pp. Moreover, $\Sigma_{\text {disc }}=\emptyset$ and $\Sigma$ coincides with the topological support of $\rho_{A}$. 
In the situation of the theorem it is also possible to calculate the distribution function of $\rho_{A}$ by a limiting procedure. Details are discussed in the literature cited above, see [LS03, LS04. Here, we mention the following results for so called finite range operators $\left(A_{\omega}\right)$ : Denote by $|\cdot|$ the number of elements of a set and by $\left.A_{\omega}\right|_{B_{n}}$ the restriction of $A_{\omega}$ to $\omega \cap B_{n}$. Define the measures $\mu_{\omega}^{n}$ on $\mathbb{R}$ by

$$
C_{0}(\mathbb{R}) \ni \varphi \mapsto \mu_{\omega}^{n}(\varphi):=\frac{1}{\left|B_{n} \cap \omega\right|} \operatorname{tr} \varphi\left(\left.A_{\omega}\right|_{B_{n}}\right) .
$$

These are just the measures associated to the eigenvalue counting functions studied so far, i.e.

$$
N_{\omega}^{n}(\lambda):=\mu_{\omega}^{n}(]-\infty, \lambda[)=\frac{\left|\left\{i: \lambda_{i}\left(\left.A_{\omega}\right|_{B_{n}}\right)<\lambda\right\}\right|}{\left|B_{n} \cap \omega\right|}
$$

with the (ordered) eigenvalues $\lambda_{i}\left(\left.A_{\omega}\right|_{B_{n}}\right)$ of $\left.A_{\omega}\right|_{B_{n}}$. Set $D:=\tau(\mathrm{Id})$. Then, the measures $\mu_{\omega}^{n}$ converge for $n \rightarrow \infty$ vaguely to the measure

$$
\varphi \mapsto \frac{1}{D} \tau(\varphi(A))=\frac{1}{D} \rho_{A}(\varphi)
$$

for almost every $\omega \in \Omega$. If $(\Omega, T)$ is uniquely ergodic, the convergence holds for all $\omega \in \Omega$. Assuming further regularity, one can even show convergence of the corresponding distribution functions with respect to the supremum norm. In any case there exists a distribution function $N_{A}$ such that $\lim _{n \rightarrow \infty} N_{\omega}^{n}(\lambda)=$ $N_{A}(\lambda)=\frac{1}{D} \rho_{A}(]-\infty, \lambda[)$ exists almost surely at all continuity points of $N_{A}$.

Note that amenability is not an issue here since the group $\mathbb{R}^{d}$ is abelian. In fact, instead of the balls $B_{n}$ we could also consider rather general van Hove sequences.

Let us finish this section by giving an explicit example of what may be called a nearest neighbour Laplacian in the context of a Delone set $\omega$. For $x \in \omega$, define the Voronoi cell $\mathcal{V}(x)$ of $x$ by

$$
\mathcal{V}(x):=\left\{p \in \mathbb{R}^{d}:\|p-x\| \leq\|p-y\| \text { for all } y \in \omega\right\} .
$$

Then, it is not hard to see that $\mathcal{V}(x)$ is a convex polytope for every $x \in \omega$. The operator $A_{\omega}$ is then defined via its matrix elements by

$$
A_{\omega}(x, y)=1 \text { if } \mathcal{V}(x) \text { and } \mathcal{V}(y) \text { share a }(d-1) \text {-dimensional face }
$$

and $A_{\omega}(x, y)=0$ otherwise.

\section{Percolation models}

In this section we shortly discuss how to fit percolation operators, more precisely site percolation operators, in our framework. In fact, edge percolation or mixed percolation could be treated along the same lines.

Theoretical physicists have been interested in Laplacians on percolation graphs as quantum mechanical Hamiltonians for quite a while dGLM59b, dGLM59a, KE72, $\mathrm{CCF}^{+} 86$. Somewhat later several computational physics 
papers where devoted to the numerical analysis of spectral properties of percolation Hamiltonians, see e.g [SAH82, KB97, KB98b, KB98a, KB02]. More recently there was a series of rigorous mathematical results on percolation models [BK01, Ves05a, Ves05b, KM, MS].

We have to identify the abstract quantities introduced in the abstract setting in the context of percolation.

A graph $G$ may be equivalently defined by its vertex set $X$ and its edge set $E$, or by its vertex set $X$ and the distance function $d: X \times X \rightarrow\{0\} \cup \mathbb{N}$. We choose the second option and tacitly identify the graph with its vertex set. In particular, each graph $X$ gives naturally rise to the so called adjacency matrix $A(X): X \times X \longrightarrow\{0,1\}$ defined by $A(X)(x, y)=1$ if $d(x, y)=1$ and $A(X)(x, y)=0$ otherwise. This adjacency matrix can be considered to be an operator on $\ell^{2}(X)$.

We assume that the vertex set of the graph is countable. Let $\Gamma$ be a group acting freely on $X$ such that the quotient $X / \Gamma$ is a finite graph, i.e. the $\Gamma$ action is quasi-transitive. The associated probability space is given by $\Omega=$ $\{0,1\}^{X}$, with the $\sigma$-field defined by the finite-dimensional cylinder sets. For simplicity let us consider only independent, identically distributed percolation on the vertices of $X$. The statements hold analogously for correlated site or bond percolation processes under appropriate ergodicity assumptions. Thus we are given a sequence of i.i.d. random variables $\omega_{x}: \Omega \rightarrow\{0,1\}$, for $x \in X$, with distribution measure $p \delta_{1}+(1-p) \delta_{0}$. The measure $\mathbb{P}$ on the probability space $\Omega$ is given by the product $\mathbb{P}=\bigotimes_{X}\left(p \delta_{1}+(1-p) \delta_{0}\right)$.

The groupoid is given by $\mathcal{G}:=\Omega \times \Gamma \ni g=(\omega, \gamma)$ and the $\mathcal{G}$-space by

$$
\mathcal{X}:=\Omega \times X \text {. }
$$

The operation of the groupoid is the same as defined in (3) and (44), the projection $\pi$ is again given by $\pi(\omega, x):=\omega$ for all $(\omega, x) \in \Omega \times X$, and the map $J$ is the same as described on page 8 for the model (RSM), where $x$ now stands for a vertex of the graph $X$.

Define for $\omega \in \Omega$ the random subset

$$
X(\omega):=\left\{x \in X \mid \omega_{x}=1\right\}
$$

of $X$. Defining $\alpha$ by $\alpha^{\omega}:=\delta_{X(\omega)}$ for all $\omega \in \Omega$ we obtain a random variable $(\mathcal{X}, \alpha)$.

Similarly as in the case of random Schrödinger operators on manifolds we define the transverse function $\nu$ on the groupoid by setting $\nu^{\omega}$ equal to the counting measure $m_{\Gamma}$ on the group for every $\omega \in \Omega$ and the $\nu$-invariant measure $\mu$ equal to $\mathbb{P}$.

We next show that we are in an admissible setting according to Definition 2.6. As for the countability condition in this definition, we proceed as follows: By the countability of the vertex set of the graph $X$ the $\sigma$-field $\mathcal{B}_{\mathcal{X}}$ is countably 
generated. Since $\mathcal{B}_{\Omega}$ is generated by the finite dimensional cylinder sets, it is countably generated as well.

Denote by $\mathcal{D}$ a fundamental domain of the covering graph $X$ and set $u_{0}(\omega, x):=\chi_{\mathcal{D}}(x)$. Then clearly

$$
\int_{\mathcal{G}^{\pi(p)}} u_{0}\left(g^{-1} p\right) d \nu^{\pi(p)}(g)=\sum_{\gamma \in \Gamma} u_{0}(\omega, \gamma x)=1 \quad \text { for all } \omega \in \Omega, x \in X .
$$

This shows the second condition of Definition 2.6.

The corresponding random operators are given by families $H_{\omega}: \ell^{2}\left(X, \alpha^{\omega}\right) \rightarrow$ $\ell^{2}\left(X, \alpha^{\omega}\right), \omega \in \Omega$, satisfying a measurability and a boundedness assumption as well as the equivariance condition

$$
H_{\omega}=U_{(\omega, \gamma)} H_{\gamma^{-1} \omega} U_{(\omega, \gamma)}^{*}
$$

for $\omega \in \Omega$ and $\gamma \in \Gamma$, where $U_{(\omega, \gamma)}: \ell^{2}\left(X, \alpha^{\gamma^{-1} \omega}\right) \rightarrow \ell^{2}\left(X, \alpha^{\omega}\right)$ is the unitary operator mapping $\phi$ to $\phi\left(\gamma^{-1} \cdot\right)$. A special example of such a random operator is given by the family $\left(A_{\omega}\right)_{\omega}$, where each $A_{\omega}$ is the adjacency matrix of the induced subgraph of $X$ generated by the vertex set $X(\omega)$.

We next show that the functional

$$
\tau(H):=\mathbb{E}\left\{\operatorname{tr}\left(M_{u_{0}} H_{\omega}\right)\right\}
$$

defined in Theorem 4.2 is a trace. As (a) of this Theorem holds by general arguments, we just have to show that the freeness condition (14) is satisfied. This can be shown as follows: Since $\Gamma$ acts freely, $\gamma x \neq x$ for all $x \in X, \gamma \in$ $\Gamma \backslash\{\epsilon\}$. Thus the set $\left\{\omega \mid \omega_{\gamma x}=\omega_{x}\right\}$ has measure smaller than one for all $x \in X$. If the graph is infinite, this poses infinitely many independent conditions on the elements in

$$
\Omega_{\gamma}:=\{\omega \mid \gamma \omega=\omega\} .
$$

Therefore, $\Omega_{\gamma}$ has measure zero for each $\gamma \in \Gamma \backslash\{\epsilon\}$. As $\Gamma$ is countable, $\bigcup_{\gamma \in \Gamma \backslash\{\epsilon\}} \Omega_{\gamma}$ has $\mathbb{P}$-measure zero as well. Thus the desired freeness statement follows.

To show that an associated operator family $\omega \mapsto H_{\omega}$ has almost surely no discrete spectrum for infinite $\Gamma$, we define the sequence of functions $f_{n}$ by

$$
f_{n}(\omega, \gamma):=\frac{1}{\left|I_{n}\right|} \chi_{I_{n}}(\gamma)
$$

Here, $I_{n}$ is an exhaustion of the infinite group $\Gamma$. The sequence $\left(f_{n}\right)$ satisfies property (21). Thus, Lemma 5.6 and Corollary 5.9 hold.

In fact, the above exhaustion and freeness properties allow us to apply Theorem 5.11 and conclude that the von Neumann algebra is of type II. More precisely

$$
\tau(\mathrm{Id})=\mathbb{E}\left\{\operatorname{tr}\left(\chi_{\mathcal{D}}\right)\right\}=p|\mathcal{D}|<\infty
$$

shows that the type is $I I_{1}$. 
Besides the discrete, essential, absolutely continuous, singular continuous, and pure point spectrum, $\sigma_{d i s c}, \sigma_{e s s}, \sigma_{a c}, \sigma_{s c}, \sigma_{p p}$, the set $\sigma_{f i n}$ consisting of eigenvalues which posses an eigenfunction with finite support is a quantity which may be associated with the whole family $H_{\omega}, \omega \in \Omega$.

The following two theorems hold for a class of percolation Hamiltonians $\left(H_{\omega}\right)_{\omega}$ which are obtained from a determinstic finite hopping range operator by a percolation process, cf. [Ves05a]. In particular the class contains the adjacency operator $\left(A_{\omega}\right)_{\omega}$ introduced above.

Recall from Section 5 that the measure $\rho_{H}$ on $\mathbb{R}$ is given by $\rho_{H}(\varphi)=\tau(\varphi(H))$ for continuous $\varphi$ on $\mathbb{R}$ with compact support.

Theorem 8.1. There exists an $\Omega^{\prime} \subset \Omega$ of full measure and subsets of the real numbers $\Sigma$ and $\Sigma_{\bullet}$, where $\bullet \in\{$ disc, ess, ac, sc,pp, fin $\}$, such that for all $\omega \in \Omega^{\prime}$

$$
\sigma\left(H_{\omega}\right)=\Sigma \quad \text { and } \quad \sigma_{\bullet}\left(H_{\omega}\right)=\Sigma \bullet
$$

for any $\bullet=$ disc, ess, ac, sc,pp, fin. Moreover, the almost-sure spectrum $\Sigma$ coincides with the topological support of $\rho_{H}$. If $\Gamma$ is infinite, $\Sigma_{\text {disc }}=\emptyset$.

If the group $\Gamma$ acting on $X$ is amenable it was shown in Ves05a that property (P5), too, holds for the family $\left(H_{\omega}\right)_{\omega}$. More precisely, it is possible to construct the measure $\rho_{H}$ by an exhaustion procedure using the finite volume eigenvalue counting functions $N_{\omega}^{n}(\lambda)$. These are defined by the formula

$$
N_{\omega}^{n}(\lambda):=\frac{\left|\left\{i \in \mathbb{N} \mid \lambda_{i}\left(H_{\omega}^{n}\right)<\lambda\right\}\right|}{\left|I_{n}\right| \cdot|\mathcal{D}|} .
$$

where $I_{n}$ is a tempered Følner sequence, $A_{n}=\bigcup_{\gamma \in I_{n}} \gamma \mathcal{D}$, and $H_{\omega}^{n}$ is the restriction of the operator $H_{\omega}$ to the space $\ell^{2}\left(X(\omega) \cap A_{n}\right)$.

Theorem 8.2. Let $\Gamma$ be amenable and $\left\{I_{n}\right\}$ be a tempered Følner sequence of $\Gamma$. Then there exists a subset $\Omega^{\prime} \subset \Omega$ of full measure and a distribution function $N_{H}$, called integrated density of states, such that for all $\omega \in \Omega^{\prime}$

$$
\lim _{n \rightarrow \infty} N_{\omega}^{n}(\lambda)=N_{H}(\lambda)
$$

at all continuity points of $N_{H} . N_{H}$ is related to the measure $\rho_{H}$ via the following trace formula

$$
N_{H}(\lambda)=\frac{\rho_{H}(]-\infty, \lambda[)}{|\mathcal{D}|} .
$$

\section{Appendix A. Some Direct integral theory}

The aim of this appendix is to prove the following lemma and to discuss some of its consequences. By standard direct integral theory Dix81, the lemma is essentially equivalent to the statement that $L^{2}(\mathcal{X}, \mu \circ \alpha)$ is canonically isomorphic to $\int_{\Omega}^{\oplus} L^{2}\left(\mathcal{X}^{\omega}, \alpha^{\omega}\right) d \mu(\omega)$.

Throughout this section let a measurable groupoid $(\mathcal{G}, \nu, \mu)$ and a random variable $(\mathcal{X}, \alpha)$ satisfying condition (6) on the associated $\mathcal{G}$-space be given. 
Lemma A.1. There exists a measurable function $N: \Omega \longrightarrow \mathbb{N}_{0} \cup\{\infty\}$, a sequence $\left(g^{(n)}\right)_{n}$ of measurable functions on $\mathcal{X}$ and a subset $\Omega^{\prime}$ of $\Omega$ of full measure satisfying the following:

- $\left(g_{\omega}^{(n)}: 1 \leq n \leq N(\omega)\right)$ is an orthonormal basis of $L^{2}\left(\mathcal{X}^{\omega}, \alpha^{\omega}\right)$ for every $\omega \in \Omega^{\prime}$.

- $g_{\omega}^{(n)}=0$ for $n>N(\omega), \omega \in \Omega^{\prime}$.

- $g_{\omega}^{(n)}=0$ for $\omega \notin \Omega^{\prime}$.

Proof. Let $\mathcal{D}$ be a countable generator of the $\sigma$-algebra of $\mathcal{X}$ such that $\mu \circ \alpha(D)<$ $\infty$ for every $D \in \mathcal{D}$. Such a $\mathcal{D}$ exists by condition 6 . Let $\left(f^{(n)}\right)_{n \in \mathbb{N}}$ be the family of characteristic functions of sets in $\mathcal{D}$. By assumption on $\mathcal{D}$, we infer that $\left(f^{(n)}\right)$ are total in $L^{2}(\mathcal{X}, \mu \circ \alpha)$.

By the Fubini Theorem, there exist a set $\Omega^{\prime}$ of full measure, such that $f_{\omega}^{(n)}$ belongs to $L^{2}\left(\mathcal{X}^{\omega}, \alpha^{\omega}\right)$ for every $n \in \mathbb{N}$ and every $\omega \in \Omega^{\prime}$. As $\mathcal{D}$ generates the $\sigma$-algebra of $\mathcal{X}$ and $\mathcal{X}^{\omega}$ is equipped with the the induced $\sigma$-algebra, we infer that $\left(f_{\omega}^{(n)}\right)_{n \in \mathbb{N}}$ is total in $L^{2}\left(\mathcal{X}, \alpha^{\omega}\right)$ for every $\omega \in \Omega^{\prime}$.

Now, define for $n \in \mathbb{N}$ the function $h^{(n)} \in L^{2}(\mathcal{X}, \mu \circ \alpha)$ by setting $h^{(n)}(p)=$ $f^{(n)}(p)$ if $\pi(p) \in \Omega^{\prime}$ and $h^{(n)}(p)=0$, otherwise.

Applying the Gram-Schmidt-orthogonalization procedure to $\left(h_{\omega}^{(n)}\right)_{n \in \mathbb{N}}$ simultaneously for all $\omega \in \Omega^{\prime}$, we find $N: \Omega \longrightarrow \mathbb{N}_{0} \cup\{\infty\}$ and $g_{\omega}^{(n)}$ as desired. (This simultaneous Gram-Schmidt procedure is a standard tool in direct integral theory, see [Dix81] for details.) The proof of the Lemma is finished.

Proposition A.2. Let $\left(A_{\omega}\right)$ be a family of bounded operators $A_{\omega}: L^{2}\left(\mathcal{X}^{\omega}, \alpha^{\omega}\right) \longrightarrow L^{2}\left(\mathcal{X}^{\omega}, \alpha^{\omega}\right)$ such that

$$
\omega \mapsto\left\langle f_{\omega}, A_{\omega} g_{\omega}\right\rangle_{\omega} \text { is measurable for arbitrary } f, g \in L^{2}(\mathcal{X}, \mu \circ \alpha) .
$$

Then, Ah: $\mathcal{X} \longrightarrow \mathbb{C},(A h)(p) \equiv\left(A_{\pi(p)} h_{\pi(p)}\right)(p)$ is measurable for every $h:$ $\mathcal{X} \longrightarrow \mathbb{C}$ measurable with $h_{\omega} \in L^{2}\left(\mathcal{X}^{\omega}, \alpha^{\omega}\right)$ for every $\omega \in \Omega$.

Proof. Let $h$ be be as in the assumption. Invoking suitable cutoff procedures, we can assume without loss of generality

$$
h \in L^{2}(\mathcal{X}, \mu \circ \alpha) \text { as well as }\left\|A_{\omega}\right\| \leq C \text { for all } \omega \in \Omega,
$$

for a suitable $C$ independent of $\omega$. Obviously, (37) implies that $\omega \mapsto\left\langle f_{\omega}, A_{\omega} g_{\omega}\right\rangle_{\omega}$ is measurable for every $f, g: \mathcal{X} \longrightarrow \mathbb{C}$ measurable with $f_{\omega}, g_{\omega} \in L^{2}\left(\mathcal{X}^{\omega}, \alpha^{\omega}\right)$ for every $\omega \in \Omega$. Thus, with $g^{(n)}, n \in \mathbb{N}$, as in the previous lemma, we see that

$$
\omega \mapsto\left\langle g_{\omega}^{(n)}, A_{\omega} h_{\omega}\right\rangle \text { is measurable for every } n \in \mathbb{N} \text {. }
$$

In particular,

$$
\mathcal{X} \longrightarrow \mathbb{C}, p \mapsto\left\langle g_{\pi(p)}^{(n)}, A_{\pi(p)} h_{\pi(p)}\right\rangle_{\pi(p)} g^{(n)}(p) \text { is measurable for every } n \in \mathbb{N} \text {. }
$$


As $\left(g_{\omega}^{(n)}: 1 \leq n \leq N(\omega)\right)$ is an orthonormal basis in $L^{2}\left(\mathcal{X}^{\omega}, \alpha^{\omega}\right)$ for every $\omega \in \Omega^{\prime}$ and $g_{\omega}^{(n)}=0$ for $n>N(\omega)$ and $\omega \in \Omega^{\prime}$, we have

$$
\left(A_{\omega} h_{\omega}\right)(p)=\sum_{n=1}^{\infty}\left\langle g_{\omega}^{(n)},\left(A_{\omega} h_{\omega}\right)\right\rangle_{\omega} g^{(n)}(p)
$$

for almost every $\omega \in \Omega$. Note that the last equality holds in the $L^{2}\left(\mathcal{X}^{\omega}, \alpha^{\omega}\right)$-sense. By (38) and the Fubini Theorem, this implies $\left(A_{\omega} h_{\omega}\right)(p)=$ $\sum_{n=1}^{\infty}\left\langle g_{\omega}^{(n)},\left(A_{\omega} h_{\omega}\right)\right\rangle_{\omega} g^{(n)}(p)$ in the sense of $L^{2}(\mathcal{X}, \mu \circ \alpha)$ and the desired measurability follows.

Proposition A.3. Let $C>0$ and $\left(A_{\omega}\right)$ be a family of operators $A_{\omega}: L^{2}\left(\mathcal{X}^{\omega}, \alpha^{\omega}\right) \longrightarrow L^{2}\left(\mathcal{X}^{\omega}, \alpha^{\omega}\right)$ with $\left\|A_{\omega}\right\| \leq C$ for every $\omega \in \Omega$ and $p \mapsto$ $\left(A_{\pi(p)} f_{\pi(p)}\right)(p)$ measurable for every $f \in L^{2}(\mathcal{X}, \mu \circ \alpha)$. Let $A: L^{2}(\mathcal{X}, \mu \circ \alpha) \longrightarrow$ $L^{2}(\mathcal{X}, \mu \circ \alpha), \quad(A f)(p)=\left(A_{\pi(p)} f_{\pi(p)}\right)(p)$ be the associated operator. Then, $A_{\omega}=0$ for $\mu$-almost every $\omega \in \Omega$ if $A=0$.

Proof. Choose $g^{(n)}, n \in \mathbb{N}$, as in Lemma A.1 and let $\mathcal{E}$ be a countable dense subset of $L^{2}(\Omega, \mu)$. For $f \in L^{2}(\mathcal{X}, \mu \circ \alpha)$ with $f(p)=g^{(n)}(p) \psi(\pi(p))$ for $n \in \mathbb{N}$ and $\psi \in \mathcal{E}$, we can then calculate

$$
0=A f=\left(p \mapsto \psi(\pi(p))\left(A_{\pi(p)} g_{\pi(p)}^{(n)}\right)(p)\right) .
$$

As $\mathcal{E}$ is dense and countable, we infer, for $\mu$-almost all $\omega \in \Omega$,

$$
A_{\omega} g_{\omega}^{(n)}=0 \text { for all } n \in \mathbb{N} \text {. }
$$

This proves the statement, as $\left(g_{\omega}^{(n)}: n \in \mathbb{N}\right)$ is total in $L^{2}\left(\mathcal{X}^{\omega}, \alpha^{\omega}\right)$ for almost every $\omega \in \Omega$.

Corollary A.4. Let $\left(A_{\omega}\right)$ and $\left(B_{\omega}\right)$ be random operators with associated operators $A$ and $B$ respectively. Then $A=B$ implies $\left(A_{\omega}\right) \sim\left(B_{\omega}\right)$.

Proof. This is immediate from the foregoing proposition.

\section{Appendix B. A Proposition from measure theory}

In this appendix we give a way to calculate the point part of a finite measure on $\mathbb{R}$. Recall that a measure is called continuous if it does not have a point part.

We start with the following Lemma.

Lemma B.1. Let $\mu$ be a continuous finite measure on $\mathbb{R}$. Then $\lim _{n \rightarrow \infty} \mu\left(I_{n}\right)=$ 0 for every sequence $\left(I_{n}\right)$ of open intervals whose lengths tend to zero.

Proof. Assume the contrary. Then there exists a sequence of open intervals $\left(I_{n}\right)$ with $\left|I_{n}\right| \rightarrow 0$ and a $\delta>0$ with $\mu\left(I_{n}\right) \geq \delta, n \in \mathbb{N}$. For each $n \in \mathbb{N}$ choose an arbitrary $x_{n} \in I_{n}$.

If the sequence $\left(x_{n}\right)$ were unbounded, one could find a subsequence $\left(I_{n_{k}}\right)_{k \in \mathbb{N}}$ of $\left(I_{n}\right)$ consisting of pairwise disjoint intervals. This would imply the contradiction $\mu(\mathbb{R}) \geq \sum_{k=1}^{\infty} \mu\left(I_{n_{k}}\right) \geq \sum_{k=1}^{\infty} \delta=\infty$. 
Thus, the sequence $\left(x_{n}\right)$ is bounded and therefore contains a converging subsequence. Without loss of generality we assume that $x_{n} \rightarrow x$ for $n \rightarrow \infty$. For every open interval $I$ containing $x$ we then have $\mu(I) \geq \mu\left(I_{n}\right)$ for $n$ large enough. This gives $\mu(I) \geq \delta$ for every such interval. From Lebesgue Theorem, we then infer $\mu(\{x\}) \geq \delta$, contradicting the continuity of $\mu$.

Proposition B.2. Let $\mu$ be a finite measure on $\mathbb{R}$ with point part $\mu^{p p}$ and continuous part $\mu^{c}$. Then, for every $B \subset \mathbb{R}, \mu^{p p}(B)$ is given by

$$
\mu^{p p}(B)=\lim _{k \rightarrow \infty} \lim _{n \rightarrow \infty} \sup _{|J| \leq n^{-1}, J \in \mathcal{J}^{k}} \mu(B \cap J) .
$$

Proof. Obviously, the limits on the right hand side of the formula make sense. We show two inequalities:

" $\leq$ ": Let $\left\{x_{i}\right\}$ be a countable subset of $\mathbb{R}$ with $\mu^{p p}=\sum_{i} \mu\left(\left\{x_{i}\right\}\right) \delta_{x_{i}}$, where $\delta_{x}$ denotes the point measure with mass one at $x$. Then, we have $\mu^{p p}(B)=$ $\sum_{x_{i} \in B} \mu\left(\left\{x_{i}\right\}\right)$. For every $\epsilon>0$, we can then find a finite subset $B_{\epsilon}$ of $\left\{x_{i}\right.$ : $\left.x_{i} \in B\right\}$ with number of elements $\# B_{\epsilon}$ and $\mu^{p p}(B) \leq \epsilon+\sum_{x \in B_{\epsilon}} \mu(\{x\})$. This easily gives

$$
\mu^{p p}(B) \leq \epsilon+\sum_{x \in B_{\epsilon}} \mu(\{x\}) \leq \epsilon+\mu(B \cap I)
$$

for suitable $J \in \mathcal{J}^{\# B_{\epsilon}}$ of arbitrary small Lebesgue measure. As $\epsilon$ is arbitrary, the desired inequality follows.

" $\geq$ ": By the foregoing lemma, we easily conclude for every $k \in \mathbb{N}$ that $\lim _{n \rightarrow \infty} \sup _{J \in \mathcal{J}^{k},|J| \leq n^{-1}} \mu^{c}(B \cap J)=0$. Combining this with the obvious inequality $\mu^{p p}(B) \geq \mu^{p p}(B \cap J)$ valid for arbitrary measurable $B, J \subset \mathbb{R}$, we infer

$$
\begin{aligned}
\mu^{p p}(B) & \geq \lim _{k \rightarrow \infty} \lim _{n \rightarrow \infty} \sup _{|J| \leq n^{-1}, J \in \mathcal{J}^{k}} \mu^{p p}(B \cap J) \\
& =\lim _{k \rightarrow \infty} \lim _{n \rightarrow \infty} \sup _{|J| \leq n^{-1}, J \in \mathcal{J}^{k}} \mu^{p p}(B \cap J)+\lim _{k \rightarrow \infty} \lim _{n \rightarrow \infty} \sup _{|J| \leq n^{-1}, J \in \mathcal{J}^{k}} \mu^{c}(B \cap J) \\
& =\lim _{k \rightarrow \infty} \lim _{n \rightarrow \infty} \sup _{|J| \leq n^{-1}, J \in \mathcal{J}^{k}} \mu(B \cap J) .
\end{aligned}
$$

This finishes the proof.

\section{ApPendix C. UniQUenESS LEMMA FOR THE LAPLACE TRANSFORM}

The results in PV01 use heavily the Laplace transform techniques developed in papers by Pastur and Šubin Pas71, Š Sub82. In the present paper only the uniqueness lemma is used. In the literature the uniqueness lemma for the Laplace transform is stated mostly for finite measures (e.g., Theorem 22.2 in [Bil95]). For the convenience of the reader we show how to adapt the uniqueness result to our case, where the distribution function $N_{H}$ is unbounded. 
Lemma C.1. Let $\left.f_{1}, f_{2}:\right] 0, \infty[\rightarrow \mathbb{R}$ be monotonously increasing function with $\lim _{\lambda \searrow 0} f_{1}(\lambda)=\lim _{\lambda \searrow 0} f_{2}(\lambda)=0$. Let the integrals

$$
\int_{0}^{\infty} e^{-t \lambda} d f_{j}(\lambda), j=1,2, t>0
$$

be finite and moreover

$$
\int_{0}^{\infty} e^{-t \lambda} d f_{1}(\lambda)=\int_{0}^{\infty} e^{-t \lambda} d f_{2}(\lambda)
$$

for all positive $t$.

Then the sets of continuity points of $f_{1}$ and $f_{2}$ coincide and for $\lambda_{0}$ in this set we have $f_{1}\left(\lambda_{0}\right)=f_{2}\left(\lambda_{0}\right)$.

Proof. Choose $s>0$ arbitrary. The measures

$$
\mu_{j}(g):=\int_{0}^{\infty} g(\lambda) e^{-s \lambda} d f_{j}(\lambda)
$$

are finite, since $\mu_{j}(1)=\int_{0}^{\infty} e^{-s \lambda} d f_{j}(\lambda)<\infty$ by assumption. Since

$$
\mu_{j}\left(e^{-t \cdot}\right)=\int_{0}^{\infty} e^{-(t+s) \lambda} d f_{j}(\lambda)=\tilde{f}_{j}(t+s),
$$

we have, by assumption, that the Laplace transforms of the measures $\mu_{j}$ coincide for all $t>0$ :

$$
\mu_{1}\left(e^{-t \cdot}\right)=\mu_{2}\left(e^{-t \cdot}\right) .
$$

As we are dealing with finite measures, the Theorem 22.2 in Bil95] implies $\mu_{1}=\mu_{2}$. We consider

$$
\mu_{j}([0, E])=\int_{0}^{E} e^{-s \lambda} d f_{j}(\lambda)
$$

as a sequence of integrals depending on the parameter $s \rightarrow 0$. Since

$$
e^{-s \cdot}:\left[0, \lambda_{0}\right] \rightarrow[0,1]
$$

converges uniformly and monotonously to the constant function 1 , we conclude by Beppo Levi's theorem

$$
\lim _{s \searrow 0} \mu_{j}\left(\left[0, \lambda_{0}\right]\right)=\int_{0}^{\lambda_{0}} d f_{j}(\lambda) .
$$

For a continuity point $\lambda_{0}$ of $f_{1}$ we have

$$
\int_{0}^{\lambda_{0}} d f_{1}(\lambda)=f_{1}\left(\lambda_{0}\right)
$$

which implies $f_{1}\left(\lambda_{0}\right)=f_{2}\left(\lambda_{0}\right)$.

Corollary C.2. Under the assumptions of the Lemma C.1 we have

$$
\int_{0}^{\infty} g(\lambda) d f_{1}(\lambda)=\int_{0}^{\infty} g(\lambda) d f_{2}(\lambda)
$$

for all continuous functions $g$ with compact support. 


\section{REFERENCES}

[AS93] T. Adachi and T. Sunada. Density of states in spectral geometry. Comment. Math. Helv., 68(3):480-493, 1993.

[AK81] M. A. Akcoglu and U. Krengel. Ergodic theorems for superadditive processes. $J$. Reine Angew. Math., 323:53-67, 1981.

[AA] S. Aubry and G. André Analyticity breaking and Anderson localization in incommensurate lattices. Ann. Isr. Phys. Soc., 3:133-164, 1980.

[AS82] J. Avron and B. Simon. Almost periodic Schrödinger operators. I. Limit periodic potentials. Comm. Math. Phys., 82(1):101-120, 1981/82.

[AS83] J. Avron and B. Simon. Almost periodic Schrödinger operators. II. The integrated density of states. Duke Math. J., 50(1):369-391, 1983.

[Bel86] J. Bellissard. K-Theory of $C^{*}$-algebras in solid state physics. In Statistical Mechanics and Field Theory: Mathematical Aspects, Eds. T.C. Dorlas, N. M. Hugenholz, M. Winnink, Lecture Notes in Physics, Vol 257, Springer, Berlin, pp. 99-156, 1986.

[Bel92] J. Bellissard. Gap labelling theorems for Schrödinger operators. In From Number Theory to Physics, Eds. M. Waldschmitt, P. Moussa, J.M. Luck, C. Itzykson, Springer, New York, pp. 538-630, 1992.

[BHZ00] J. Bellissard, D. J. L. Herrmann, M. Zarrouati. Hulls of aperiodic solids and gap labelling theorems. In Directions in mathematical quasicrystals, Eds. M. Baake, R. V. Moody, CRM Monograph Series 13, Amer. Math. Soc., Providence, RI, pp. 207$258,2000$.

[BLT86] J. Bellissard, R. Lima, D. Testard. Almost periodic Schrödinger operators. In Mathematics + Physics Vol. 1, Ed. L. Streit, World Scientific, Singapore, pp. 1-64, 1985.

[BT82] J. Bellissard and D. Testard, Quasi-periodic Hamiltonians. A mathematical approach. Proc. Sympos. Pure Math. 38:579-582, 1982.

[BKL01] J. Bellissard, J. Kellendonk, A. Legrand Gap-labelling for three-dimensional aperiodic solids. C. R. Acad. Sci. Paris Sr. I Math. 332 (2001), no. 6, 521-525.

[BOH01] M.-T. Benameur, H. Oyono-Oyono Gap-labelling for quasi-crystals (proving a conjecture by J. Bellissard) Operator algebras and mathematical physics (Constanţa, 2001), 11-22, Theta, Bucharest, 2003.

[BBG01] J. Bellissard, R. Benedetti, and J.-M. Gamdaudo Spaces of tilings, finite telescopic approximations, and gap-labeling Comm. Math. Phys., 261(1):1-41, 2006

[BK01] M. Biskup, W. König, Long-time tails in the parabolic Anderson model with bounded potential Ann. Probab. 29(2):636-682, 2001.

[Bil95] P. Billingsley. Probability and measure. John Wiley \& Sons Inc., New York, third edition, 1995.

[Cha84] I. Chavel. Eigenvalues in Riemannian geometry. Academic Press Inc., Orlando, FL, 1984.

$\left[\mathrm{CCF}^{+} 86\right]$ J. T. Chayes, L. Chayes, J. R. Franz, J. P. Sethna, and S. A. Trugman. On the density of states for the quantum percolation problem. J. Phys. A, 19(18):L1173L1177, 1986.

[Cho92] W. Chojnacki. A generalized spectral duality theorem. Comm. Math. Phys., 143(3):527-544, 1992.

[Car86] R. Carmona. Random Schrödinger operators, in: Lecture notes in Mathematics, 1180 Springer, Berlin, 1986.

[CL90] R. Carmona, J. Lacroix. Spectral theory of random Schrödinger operators. Birkhäuser Boston Inc., Boston, MA, 1990.

[CMS73] L. A. Coburn, R. D. Moyer, I. M. Singer. $C^{*}$-algebras of almost periodic pseudodifferential operators. Acta Math., 130:279-307, 1973. 
[Con79] A. Connes. Sur la théorie non commutative de l'intégration. In Algèbres d'opérateurs (Sém., Les Plans-sur-Bex, 1978), pages 19-143. Springer, Berlin, 1979.

[Con94] A. Connes. Noncommutative geometry. Academic Press, San Diego, 1994.

[CFSK87] H.L. Cycon, R. G. Froese, W. Kirsch, B. Simon. Schrödinger operators with application to quantum mechanics and global geometry. Texts and Monographs in Physics, Spinger, Berlin, 1987.

[dGLM59a] P.-G. de Gennes, P. Lafore, and J. Millot. Amas accidentels dans les solutions solides désordonnées. J. of Phys. and Chem. of Solids, 11(1-2):105-110, 1959.

[dGLM59b] P.-G. de Gennes, P. Lafore, and J. Millot. Sur un phénomène de propagation dans un milieu désordonné. J. Phys. Rad., 20:624, 1959.

[Dix77] J. Dixmier. $C^{*}$-algebras. North-Holland Publishing Co., Amsterdam, 1977.

[Dix81] J. Dixmier. Von Neumann algebras. North-Holland Publishing Co., Amsterdam, 1981.

[DLMSY03] J. Dodziuk, P. Linnell, V. Mathai, T. Schick, S. Yates. Approximating $L^{2}$ invariants and the Atiyah Conjecture. Comm.Pure Appl. Math., 56(7):839-873, 2003.

[DM97] J. Dodziuk, V. Mathai. Approximating $L^{2}$ invariants of amenable covering spaces: a heat kernel approach, in: In: Lipa's Legacy, Contemp. Math., 211:151-167, 1997. Amer. Math. Soc., Providence.

[DM98] J. Dodziuk, V. Mathai. Approximating $L^{2}$ invariants of amenable covering spaces: a combinatorial approach. J. Funct. Anal., 154(2):359-378, 1998.

[FN77] M. Fukushima, S. Nakao. On spectra of the Schrödinger operator with a white Gaussian noise potential. Z. Wahrscheinlichkeitstheorie und Verw. Gebiete, 37(3):267-274, $1976 / 77$.

[FNN75] M. Fukushima, H. Nagai, S. Nakao. On an asymptotic property of spectra of a random difference operator. Proc. Japan Acad., 51:100-102, 1975.

[Fuk74] M. Fukushima. On the spectral distribution of a disordered system and the range of a random walk. Osaka J. Math., 11:73-85, 1974.

[Fuk81] M. Fukushima. On asymptotics of spectra of Schrödinger operators. In Statistical and physical aspects of Gaussian processes (Saint-Flour, 1980), pages 335-347. CNRS, Paris, 1981.

[GS79] I. I. Gihman and A. V. Skorohod. Theory of Stochastic Processes, vols. I-III. Springer, Berlin, 74-79.

[GJLS99] A. Y. Gordon, S. Jitomirskaya, Y. Last, B. Simon. Duality and singular continuous spectrum in the almost Mathieu equation. Acta. Math., 178(2):169-183, 1997.

[Hof93] A. Hof. Some Remarks on Discrete Aperiodic Schrödinger Operators. J. stat. Phys., 72(5/6):1353-1374, 1993.

[Hof95] A. Hof. A Remark on Schrödinger Operators on Aperiodic Tilings. J. stat. Phys., 81(3/4):851-855, 1995.

[KX87] J. Kaminker, J. Xia. The spectrum of operators elliptic along the orbits of $\mathbf{R}^{n}$ actions. Comm. Math. Phys., 110(3):427-438, 1987.

[KP03] J. Kaminker and I. Putnam A proof of the gap labeling conjecture Michigan Math. J. 51 (2003), no. 3, 537-546.

[KB97] J. W. Kantelhardt and A. Bunde. Electrons and fractons on percolation structures at criticality: Sublocalization and superlocalization. Phys. Rev. E, 56:6693-6701, 1997.

[KB98a] J. W. Kantelhardt and A. Bunde. Extended fractons and localized phonons on percolation clusters. Phys. Rev. Lett., 81:4907-4910, 1998.

[KB98b] J. W. Kantelhardt and A. Bunde. Wave functions in the Anderson model and in the quantum percolation model: a comparison. Ann. Phys. (8), 7(5-6):400-405, 1998.

[KB02] J. W. Kantelhardt and A. Bunde. Sublocalization, superlocalization, and violation of standard single-parameter scaling in the Anderson model. Phys. Rev. B, 66, 2002. 
[KE72] S. Kirkpatrick and T. P. Eggarter. Localized states of a binary alloy. Phys. Rev. B, 6:3598, 1972.

[Kel95] J. Kellendonk. Noncommutative geometry of tilings and gap labelling. Rev. Math. Phys., 7(7):1133-1180, 1995.

[Kel97] J. Kellendonk. The local structure of tilings and their integer group of coinvariants. Comm. Math. Phys., 187:115-157, 1997.

[Kir81] W. Kirsch. Über Spektren stochastischer Schrödingeroperatoren. Dissertation, Ruhr-Universität Bochum, 1981.

[Kir85] W. Kirsch. On a class of random Schrödinger operators. Adv. in Appl. Math., 6(2):177-187, 1985.

[Kir89] W. Kirsch. Random Schrödinger operators. In Schrödinger Operators, Eds. H. Holden, A. Jensen, Lecture Notes in Physics, Vol 345, Springer, Berlin, 1989.

[KM82a] W. Kirsch, F. Martinelli. On the density of states of Schrödinger operators with a random potential. J. Phys. A, 15(7):2139-2156, 1982.

[KM82b] W. Kirsch, F. Martinelli. On the ergodic properties of the spectrum of general random operators. J. Reine Angew. Math., 334:141-156, 1982.

[KM82c] W. Kirsch, F. Martinelli. On the spectrum of Schrödinger operators with a random potential. Comm. Math. Phys., 85(3):329-350, 1982.

$[\mathrm{KM}] \quad$ W. Kirsch and P. Müller. Spectral properties of the laplacian on bond-percolation graphs. http://arXiv.org/math-ph/0407047, to appear in Math. Z.

[Kot77] S. Kotani. On asymptotic behaviour of the spectra of a one-dimensional Hamiltonian with a certain random coefficient. Publ. Res. Inst. Math. Sci., 12(2):447-492, $1976 / 77$.

[Kor95] Y. A. Kordyukov. Functional calculus for tangentially elliptic operators on foliated manifolds. in Analysis and geometry in foliated manifolds (Santiago de Compostela, 1994), pages 113-136, World Sci. Publishing, River Edge, NJ, 1995.

[KS79] H. Kunz, B. Souillard. Sur le spectre des opératerus aux différences finies aléatoires. Commun. Math. Phys., 78:201-46, 1979.

[Len99] D. H. Lenz. Random operators and crossed products. Math. Phys. Anal. Geom., 2(2):197-220, 1999.

[LPV04] D. H. Lenz, N. Peyerimhoff, and I. Veselić. Integrated density of states for random metrics on manifolds. Proc. London Math. Soc. 88 (2004), 733-752

[LPV03] D. Lenz, N. Peyerimhoff, and I. Veselić. Random Schrödinger operators on manifolds. http://arxiv.org/math-ph/0212057 Markov Process. Related Fields, 9(4):717-728, 2003.

[LS01] D. H. Lenz, P. Stollmann. Quasicrystals, aperiodic order, and groupoid von Neumann algebras. C. R. Acad. Sci. Ser. I, 334:1131-1136, 2002.

[LS03] D. H. Lenz, P. Stollmann. Algebras of random operators associated to Delone dynamical systems Mathematical Physics, Analysis and Geometry 6 (2003), 269-290.

[LS04] D. H. Lenz, P. Stollmann. An ergodic theorem for Delone dynamical systems and existence of the density of states, J. Anal. Math., to appear

[LV] D. Lenz, I. Veselić. Hamiltonians on discrete structures: jumps of the integrated density of states. in perparation.

[Lin01] E. Lindenstrauss. Pointwise theorems for amenable groups. Invent. Math., 146(2):259-295, 2001.

[MSY03] V. Mathai, T. Schick, S. Yates. Approximating spectral invariants of harper operators on graphs. II. Proc. Amer. Math. Soc., 131(6):1917-1923, 2003.

[MY02] V. Mathai, S. Yates. Approximating spectral invariants of harper operators on graphs. J. Funct. Anal., 188(1):111-136, 2002.

[MS] P. Müller and P. Stollmann. Spectral asymptotics of the laplacian on supercritical bond-percolation graphs. http://arxiv.org/math-ph/0506053. 
[Nak77] S. Nakao. On the spectral distribution of the Schrödinger operator with random potential. Japan. J. Math. (N.S.), 3(1):111-139, 1977.

[Pas71] L. A. Pastur. Selfaverageability of the number of states of the Schrödinger equation with a random potential. Mat. Fiz. i Funkcional. Anal., (Vyp. 2):111-116, 238, 1971.

[Pas72] L. A. Pastur. The distribution of eigenvalues of the Schrödinger equation with a random potential. Funkcional. Anal. i Priložen., 6(2):93-94, 1972.

[Pas74] L. A. Pastur. The distribution of the eigenvalues of Schrödinger's equation with a random potential. In Mathematical physics and functional analysis, No. V (Russian), pages 141-143, 158. Akad. Nauk Ukrain. SSR Fiz.- Tehn. Inst. Nizkih Temperatur, Kharkov, 1974.

[Pas80] L. A. Pastur. Spectral properties of disordered systems in the one-body approximation. Commun. Math. Phys., 75:179-196, 1980.

[PF92] L.A. Pastur, A. Figotin. Spectra of random and almost-periodic operators. SpringerVerlag, Berlin, 1992.

[PV01] N. Peyerimhoff, I. Veselić. Integrated density of states for ergodic random Schrödinger operators on manifolds. Geom. Dedicata 91 (1): 117-135, 2002.

[Ra91] J. G. Ratcliffe. Foundations of Hyperbolic Manifolds. Springer Verlag, 1991.

[RS78] M. Reed, B. Simon. Methods of Modern Mathematical Physics IV, Analysis of Operators. Academic Press, San Diego, 1978.

[Ren80] J. Renault. A groupoid approach to $C^{*}$-algebras. Lecture Notes in Math., 793, Springer, Berlin, 1980.

[SchY94] R. Schoen, S.-T. Yau. Lectures on differential geometry. International Press, Cambridge, MA, 1994.

[SAH82] Y. Shapir, A. Aharony, and A. B. Harris. Localization and quantum percolation. Phys. Rev. Lett., 49(7):486-489, 1982.

[Shu88] A. Shulman. Maximal ergodic theorems on groups. Dep. Lit. NIINTI, 2184, 1988.

[Šub79] M. A. Šubin. Spectral theory and the index of elliptic operators with almost-periodic coefficients. Russian Math. Surveys, 34:109-158, 1979.

[Šub82] M. A. Šubin. Density of states of self adjoint operators with almost periodic coefficients. Amer. Math. Soc. Translations, 118:307-339, 1982.

[Sto01] P. Stollmann. Caught by Disorder, Bound States in Random Media. Progress in Mathematical Physics, Vol 20, Birkhäuser, Boston, 2001.

[Szn89] A.-S. Sznitman. Lifschitz tail and Wiener sausage on hyperbolic space. Comm. Pure Appl. Math., 42(8):1033-1065, 1989.

[Szn90] A.-S. Sznitman. Lifschitz tail on hyperbolic space: Neumann conditions. Comm. Pure Appl. Math., 43(1):1-30, 1990.

[Ves04] I. Veselić. Integrated density of states and Wegner estimates for random Schrödinger operators. Contemp. Math., 340:98-184. Amer. Math. Soc., Providence, RI, 2004. arXiv.org/math-ph/0307062.

[Ves05a] I. Veselić. Quantum site percolation on amenable graphs. In Proceedings of the Conference on Applied Mathematics and Scientific Computing, pages 317-328, Dordrecht, 2005. Springer. http://arXiv.org/math-ph/0308041

[Ves05b] I. Veselić. Spectral analysis of percolation Hamiltonians. Math. Ann., 331(4):841865, 2005. http://arXiv.org/math-ph/0405006

[Wei80] J. Weidmann. Linear Operators in Hilbert Spaces. Grad. Texts in Math. 68, Springer, New York, 1980.

(D. Lenz \& I. Veselić) Fakultät für Mathematik, D-09107 TU Chemnitz, Germany $U R L:$ www.tu-chemnitz.de/mathematik/mathematische_physik/

$U R L:$ ww.tu-chemnitz.de/mathematik/schroedinger/ 
(N. Peyerimhoff) Dept. of Mathematical Sciences, Durham University, UK URL: http://www.maths.dur.ac.uk/ dmaOnp/ 